\title{
REGULARITY OF DISPLACEMENT SOLUTIONS IN HENCKY PLASTICITY. II: THE MAIN RESULT
}

Abstract. The aim of this paper is to study the problem of regularity of displacement solutions in Hencky plasticity. Here, a non-homogeneous material is considered, where the elastic-plastic properties change discontinuously. In the first part, we have found the extremal relation between the displacement formulation defined on the space of bounded deformation and the stress formulation of the variational problem in Hencky plasticity.

In the second part, we prove that the displacement solution belongs to the appropriate Sobolev space (if the stress solution belongs to the interior of a set of admissible stresses, at each point). Then we deduce a regularity theorem for displacement solutions in composite materials.

1. Introduction. The principal aim of this contribution is to prove the regularity of displacement solutions in Hencky plasticity. Here, a nonhomogeneous body is considered whose elastic-plastic properties change discontinuously.

The regularity of displacement solutions is investigated in [10] for an isotropic Hencky material with the von Mises yield criterion. The elasticplastic problems with the Tresca yield criterion or the yield criterion of soil material are not investigated. Moreover, the authors of [10] do not consider bodies clamped on the boundary.

Anzellotti and Giaquinta [1] study functionals defined on the space $B V(\Omega)$. They obtain the regularity of the minimizers under the assumption that the normal integrand $\Omega \times \mathbb{R}^{n \times m} \ni(x, \mathbf{p}) \mapsto j(x, \mathbf{p})$ is of class $C^{2}$ with respect to $\mathbf{p}$, and is continuous with respect to the first variable.

2010 Mathematics Subject Classification: Primary 49N60; Secondary 49J45, 49K30, 74C05. Key words and phrases: regularity of displacement solutions in Hencky plasticity, bounded deformation, boundary transmission problems in plasticity, composite materials. 
In [6, 12, 14 the authors study the regularity of solutions of quasi-linear (or linear) elliptic boundary transmission problems in a domain $\Omega$ (composed of a finite family of regular subdomains $\Omega_{i}$ ).

In the first part of this paper, we have found the extremal relation between the displacement formulation (defined on $B D(\Omega)$ ) and the stress formulation of the variational problems in Hencky plasticity (cf. [4]). In the second part, we prove that the displacement solution belongs to the space $L D(\Omega)$ (if the stress solution belongs to the interior of a set of admissible stresses, at each point). Moreover, under the above mentioned assumption, the relaxed Dirichlet condition is satisfied exactly by the displacement solution.

We consider all the standard yield criteria (von Mises, Tresca or the yield criterion of soil material). However, we have to assume that the stress solution belongs to the space $W^{n}(\Omega$, div) (see (2.4)).

We do not assume the continuity of the displacement field on the interface between subdomains, because the space $B D(\Omega)$ contains discontinuous functions (see (2.2)). The elastic-plastic potential is a normal integrand (see [8. Chapter 8, p. 232] and Definition 1), so it is a discontinuous function with respect to the space variable for the case of a non-homogenized body (composed of a few components). Moreover, the yield criterion may change in a discontinuous way, i.e., it may jump on the interface between subdomains.

The study of the regularity of displacement solutions is significant for the understanding of appearance of cracks.

2. Some basic definitions and theorems. Let $\Omega \subset \subset \Omega_{1}$ be bounded, open $(\Omega=\operatorname{int} \Omega)$, connected sets of class $C^{1}$ in $\mathbb{R}^{n}$.

We define the following Banach spaces (see [11], [16], [17]):

$$
\begin{gathered}
L D(\Omega) \equiv\left\{\mathbf{u} \in L^{1}(\Omega)^{n} \mid 2 \varepsilon_{i j}(\mathbf{u}) \equiv\left(\partial u_{i} / \partial x_{j}+\partial u_{j} / \partial x_{i}\right) \in L^{1}(\Omega),\right. \\
i, j=1, \ldots, n\} \\
B D(\Omega) \equiv\left\{\mathbf{u} \in L^{1}(\Omega)^{n} \mid \varepsilon_{i j}(\mathbf{u}) \in \mathbb{M}_{b}(\Omega), i, j=1, \ldots, n\right\}
\end{gathered}
$$

with the natural norms

$$
\|\mathbf{u}\|_{L D}=\|\mathbf{u}\|_{L^{1}}+\sum_{i, j}^{n}\left\|\varepsilon_{i j}(\mathbf{u})\right\|_{L^{1}},\|\mathbf{u}\|_{B D}=\|\mathbf{u}\|_{L^{1}}+\sum_{i, j}^{n}\left\|\varepsilon_{i j}(\mathbf{u})\right\|_{\mathbb{M}_{b}}
$$

There exists a continuous surjective linear trace $\gamma_{B}$ from $\left[B D(\Omega),\|\cdot\|_{B D}\right]$ into $\left[L^{1}(\operatorname{Fr} \Omega)^{n},\|\cdot\|_{L^{1}}\right]$ such that $\gamma_{B}(\mathbf{u})=\mathbf{u}_{\mid \operatorname{Fr} \Omega}$ for all $\mathbf{u} \in B D \cap C(\bar{\Omega})^{n}$ (see [16]).

A net (generalized sequence) $\left\{\mathbf{u}_{\delta}\right\}_{\delta \in D} \subset B D(\Omega)$ is convergent to $\mathbf{u}_{0} \in$ $B D(\Omega)$ in the weak* $B D$ topology if $\int_{\Omega} \mathbf{g} \cdot\left(\mathbf{u}_{0}-\mathbf{u}_{\delta}\right) d x+\int_{\Omega} \mathbf{h}: \varepsilon\left(\mathbf{u}_{0}-\mathbf{u}_{\delta}\right) \rightarrow 0$ for all $(\mathbf{g}, \mathbf{h}) \in C_{c}\left(\Omega, \mathbb{R}^{n}\right) \times C_{c}\left(\Omega, \mathbb{E}_{s}^{n}\right)$ (see [9, pp. 73-81]). The trace $\gamma_{B}$ is not 
continuous on $\left[B D(\Omega)\right.$, weak* topology] if the space $L^{1}(\operatorname{Fr} \Omega)^{n}$ is endowed with a Hausdorff topology (or a $T_{1}$-topology, see [2], [9], [16]).

In this paper we define the Banach space of measurable functions

$$
W^{n}(\Omega, \operatorname{div}) \equiv\left\{\boldsymbol{\sigma} \in L^{\infty}\left(\Omega, \mathbb{E}_{s}^{n}\right) \mid \operatorname{div} \boldsymbol{\sigma} \in L^{n}(\Omega)^{n}\right\}
$$

endowed with the natural norm $\|\boldsymbol{\sigma}\|_{W^{n}(\Omega, \operatorname{div})}=\|\boldsymbol{\sigma}\|_{L^{\infty}\left(\Omega, \mathbb{E}_{s}^{n}\right)}+\|\operatorname{div} \boldsymbol{\sigma}\|_{L^{n}(\Omega)^{n}}$ (see [16, Chapter 2, Section 7] and [2]).

Assumption 1 (cf. [2]). Let $\mathcal{K}: \bar{\Omega} \rightarrow 2^{\mathbb{E}_{s}^{n}}$ be a multifunction such that for all $x \in \bar{\Omega}, \mathcal{K}(x)$ is a convex closed subset of $\mathbb{E}_{s}^{n}$ and:

(i) if $\mathbf{z}(x) \in \mathcal{K}(x)$ for $d x$-almost every $x \in \Omega, \mathbf{z} \in C\left(\bar{\Omega}, \mathbb{E}_{s}^{n}\right)$ and $\mathbf{z}_{\text {int } \Omega} \in$ $W^{n}(\Omega, \operatorname{div})$, then $\mathbf{z}(y) \in \mathcal{K}(y)$ for every $y \in \bar{\Omega}$;

(ii) for every $y \in \bar{\Omega}$ and every $\mathbf{w} \in \mathcal{K}(y)$ there exists $\mathbf{z} \in C\left(\bar{\Omega}, \mathbb{E}_{s}^{n}\right)$ such that $\mathbf{z}_{\mid \operatorname{int} \Omega} \in W^{n}(\Omega, \operatorname{div}), \mathbf{z}(y)=\mathbf{w}$ and $\mathbf{z}(x) \in \mathcal{K}(x)$ for every $x \in \bar{\Omega}$.

Definition 1. A function $j^{*}: \Omega \times \mathbb{E}_{s}^{n} \rightarrow \mathbb{R} \cup\{\infty\}$ is called a convex normal integrand if

(i) $\mathbb{E}_{s}^{n} \ni \mathbf{w}^{*} \mapsto j^{*}\left(x, \mathbf{w}^{*}\right)$ is convex and l.s.c. for $d x$-a.e. $x \in \Omega$,

(ii) there exists a Borel function $\widetilde{j^{*}}: \Omega \times \mathbb{E}_{s}^{n} \rightarrow \mathbb{R} \cup\{\infty\}$ such that $\widetilde{j^{*}}(x, \cdot)=j^{*}(x, \cdot)$ for $d x$-a.e. $x \in \Omega($ cf. [8, Chapter 8, p. 232]).

Moreover, let

$$
\mathcal{K}(x)=\left\{\mathbf{w}^{*} \in \mathbb{E}_{s}^{n} \mid j^{*}\left(x, \mathbf{w}^{*}\right)<\infty\right\} \quad \text { for } d x \text {-a.e. } x \in \Omega .
$$

Assumption 2. There exist $k, r_{1}>0$ such that $j^{*}\left(x, \mathbf{w}^{*}\right) \leq k$ for every $\mathbf{w}^{*} \in B_{\mathbb{E}_{s}^{n}}\left(0, r_{1}\right)$ and $d x$-a.e. $x \in \Omega$, and $j^{*}$ is non-negative on $\Omega \times \mathbb{E}_{s}^{n}$. Moreover, for every $\widehat{r}>0$ there exists $c_{\widehat{r}}$ such that

$$
\begin{array}{r}
\sup \left\{\int_{\Omega} j^{*}\left(x, \mathbf{z}^{*}\right) d x \mid \mathbf{z}^{*} \in L^{\infty}\left(\Omega, \mathbb{E}_{s}^{n}\right),\left\|\mathbf{z}^{*}\right\|_{L^{\infty}}<\widehat{r}\right. \\
\text { and } \left.\mathbf{z}^{*}(x) \in \mathcal{K}(x) \text { for } d x \text {-a.e. } x \in \Omega\right\}<c_{\widehat{r}}<\infty .
\end{array}
$$

That is, the dual elastic potential $\mathbf{z}^{*} \mapsto \int_{\Omega} j^{*}\left(x, \mathbf{z}^{*}\right) d x$ is finite for every $\mathbf{z}^{*} \in L^{\infty}\left(\Omega, \mathbb{E}_{s}^{n}\right)$ that is an admissible stress field.

We consider an elastic-perfectly plastic body, occupying the given set $\Omega$, with the elasticity convex domain $\mathcal{K}(x)$ (at all $x \in \Omega$ ). We define

$$
j(x, \mathbf{w}) \equiv j^{* *}(x, \mathbf{w}) \equiv \sup \left\{\mathbf{w}: \mathbf{w}^{*}-j^{*}\left(x, \mathbf{w}^{*}\right) \mid \mathbf{w}^{*} \in \mathbb{E}_{s}^{n}\right\}
$$

for $d x$-a.e. $x \in \Omega$ and for all $\mathbf{w} \in \mathbb{E}_{s}^{n}$. This function $j$ is a convex normal integrand (cf. [8]). Let $j_{\infty}: \bar{\Omega} \times \mathbb{E}_{s}^{n} \rightarrow \mathbb{R} \cup\{\infty\}$ be defined by

$$
j_{\infty}(x, \mathbf{w}) \equiv \sup \left\{\mathbf{w}: \mathbf{w}^{*}-I_{\mathcal{K}(x)}\left(\mathbf{w}^{*}\right) \mid \mathbf{w}^{*} \in \mathbb{E}_{s}^{n}\right\}
$$


for all $x \in \bar{\Omega}$ and $\mathbf{w} \in \mathbb{E}_{s}^{n}$. Let $\mathbf{f} \in L^{n}(\Omega)^{n}$ and $\mathbf{g} \in L^{\infty}\left(\Gamma_{1}\right)^{n}$ be the volume and boundary forces. We consider the functional of total energy

$$
B D(\Omega) \ni \mathbf{u} \mapsto\left\lceil P_{\lambda, j}\right\rceil(\mathbf{u}) \equiv F_{\lambda}(\mathbf{u})+G_{j}(\varepsilon(\mathbf{u})),
$$

where

$$
F_{\lambda}(\mathbf{u}) \equiv-\lambda L(\mathbf{u})+I_{C_{a}\left(\mathbf{u}^{0}\right)}(\mathbf{u}), \quad L(\mathbf{u}) \equiv \int_{\Omega} \mathbf{f} \cdot \mathbf{u} d x+\int_{\Gamma_{1}} \mathbf{g} \cdot \gamma_{B}(\mathbf{u}) d s,
$$

and $C_{a}\left(\mathbf{u}^{0}\right)$ is the set of kinematically admissible displacements,

$$
C_{a}\left(\mathbf{u}^{0}\right) \equiv\left\{\mathbf{u} \in B D(\Omega) \mid \boldsymbol{\gamma}_{B}(\mathbf{u})_{\mid \Gamma_{0}}=\mathbf{u}^{0} \text { on } \Gamma_{0}, \mathbf{u}^{0} \in L^{1}\left(\Gamma_{0}\right)^{n}\right\} .
$$

The elastic-plastic energy $G_{j}: \mathbb{M}_{b}\left(\Omega, \mathbb{E}_{s}^{n}\right) \rightarrow \mathbb{R} \cup\{\infty\}$ is given by $G_{j}(\boldsymbol{\mu}) \equiv$ $\int_{\Omega} j(x, \boldsymbol{\mu}) d x$ if $\boldsymbol{\mu} \in L^{1}\left(\Omega, \mathbb{E}_{s}^{n}\right)$ (i.e., $\boldsymbol{\mu}$ is absolutely continuous with respect to $d x)$ and $G_{j}(\boldsymbol{\mu}) \equiv \infty$ otherwise.

Formula (2.9) describes the total elastic-perfectly plastic energy of a body occupying the given subset $\Omega$ of the space $\mathbb{R}^{n}$. The constant $\lambda \geq 0(\lambda<\infty)$ is the load multiplier (see [16, Chapter 1, Section 4]).

Assumption 3. Let $\Gamma_{1}=\operatorname{Fr} \Omega \cap \mathcal{C}$, where $\mathcal{C}=\operatorname{cl} \operatorname{int} \mathcal{C} \subset \Omega_{1}$ is a closed Caccioppoli set and $d s(\operatorname{Fr} \Omega \cap \operatorname{Fr} \mathcal{C})=0$ (cf. [4, (5.3)]).

3. Regularity of displacement solutions. In this section we state our main result that the displacement solution belongs to the space $L D(\Omega)$ (if the stress solution belongs to the interior of a set of admissible stresses, at each point). The proof is given in Section 5 . Here, $\mathbf{u}^{0}=\mathbf{0}$ on $\Gamma_{0}$ is assumed. Moreover, it is not assumed that the set $\mathcal{K}(x)$ is bounded for any $x \in \bar{\Omega}$.

The original problem $\left(P_{\lambda, j}\right)$ defined in [4, (6.5)], where $\mathbf{u}^{0}=\mathbf{0}$ on $\Gamma_{0}$, is connected with the limit analysis problem $\left(P_{0, j}\right)_{A L}$ :

$$
\begin{aligned}
& \left(P_{0, j}\right)_{A L} \text { find } \inf \left\{\int_{\Omega} j_{\infty}(x, \varepsilon(\mathbf{u})) d x \mid \mathbf{u} \in L D(\Omega),\right. \\
& \left.\qquad \gamma_{B}(\mathbf{u})=\mathbf{0} \text { on } \Gamma_{0}, L(\mathbf{u})=1\right\}
\end{aligned}
$$

(see (2.8), (2.10) and [4, (3.13)]). The formula $\left(P_{\lambda, j}\right)$ describes the total elastic-perfectly plastic energy of a body occupying $\Omega$. The limit analysis problem $\left(P_{0, j}\right)_{A L}$ is significant for the study of coercivity of the elasticperfectly plastic energy $\left(P_{\lambda, j}\right)$ (see Proposition 14). The bidual relaxed problem $\left(R P_{\lambda, j}^{* *}\right)$ defined in [4, (4.16)] with $\mathbf{u}^{0}=\mathbf{0}$ on $\Gamma_{0}$ is connected with the limit analysis problem 


$$
\begin{aligned}
\left(R P_{0, j}^{* *}\right)_{A L} & \text { find } \inf \left\{\int_{\Gamma_{0}} j_{\infty}\left(x,-\gamma_{B}(\mathbf{u}) \otimes_{s} \boldsymbol{\nu}\right) d s+\int_{\Omega} j_{\infty}\left(x, \boldsymbol{\varepsilon}(\mathbf{u})_{a}\right) d x\right. \\
& \left.+\int_{\Omega} j_{\infty}\left(x, \frac{d \boldsymbol{\varepsilon}(\mathbf{u})_{s}}{d\left|\varepsilon(\mathbf{u})_{s}\right|}\right) d\left|\boldsymbol{\varepsilon}(\mathbf{u})_{s}\right| \mid \mathbf{u} \in B D(\Omega), L(\mathbf{u})=1\right\}
\end{aligned}
$$

The functional $\left\lceil P_{\lambda, j}^{*}\right\rceil$ is defined by

$$
W^{n}(\Omega, \operatorname{div}) \ni \boldsymbol{\sigma} \mapsto\left\lceil P_{\lambda, j}^{*}\right\rceil(\boldsymbol{\sigma})=-\left(F_{\lambda, 1}\right)^{*}\left(-\varepsilon^{*}(\boldsymbol{\sigma})\right)-G_{1, j}^{*}(\boldsymbol{\sigma}),
$$

where $\left(F_{\lambda, 1}\right)^{*}$ and $G_{1, j}^{*}$ are given in [4, (6.7) and (6.8)].

A maximizer of $\left\lceil P_{\lambda, j}^{*}\right\rceil$ is a solution of the stress problem. Similarly, a minimizer of $\left(R P_{\lambda, j}^{* *}\right)$ is a solution of the relaxed displacement problem. Due to [4, Lemma 13 and Proposition 25] the dual problem (given by $\left\lceil P_{\lambda, j}^{*}\right\rceil$ ) and the relaxed dual problem $\left(R P_{\lambda, j}^{*}\right)$ (cf. [4. (4.15), (4.10), (4.13) and (7.62)]) are equivalent.

Assumption 4. There exist $\lambda_{r}>0$ and $\boldsymbol{\sigma}_{\lambda_{r}} \in C\left(\bar{\Omega}, \mathbb{E}_{s}^{n}\right) \cap W^{n}(\Omega$, div $)$ such that $\boldsymbol{\beta}_{B}\left(\boldsymbol{\sigma}_{\lambda_{r}}\right)=\lambda_{r} \mathbf{g}$ on $\Gamma_{1}$ and $\boldsymbol{\sigma}_{\lambda_{r}}(x) \in \mathcal{K}(x)$ for every $x \in \Omega$. Moreover, let $L(\mathbf{u})=L(\mathbf{u}+\overline{\mathbf{u}})$ for every $\mathbf{u} \in L D(\Omega)$ and $\overline{\mathbf{u}} \in L D(\Omega)$ with $\varepsilon(\overline{\mathbf{u}})=\mathbf{0}$ in $\Omega$, if $d s\left(\Gamma_{0}\right)=0$ and $\inf \left(P_{0, j}\right)_{A L}=\infty$.

By Assumption 4 the boundary force $\mathbf{g} \in L^{\infty}\left(\Gamma_{1}\right)^{n}$ is a regular function.

We define the function $\left\lceil R P_{\lambda, j}^{* *}\right\rceil: B D(\Omega) \rightarrow \mathbb{R} \cup\{+\infty\}$ by

$$
\left\lceil R P_{\lambda, j}^{* *}\right\rceil(\mathbf{u})=\left(F_{\lambda, R}\right)^{* *}(\mathbf{u})+G_{j}^{* *}(\varepsilon(\mathbf{u})),
$$

where

$$
\begin{aligned}
\left(F_{\lambda, R}\right)^{* *}(\mathbf{u}) \equiv & -\lambda\left(\int_{\Omega} \mathbf{f} \cdot \mathbf{u} d x+\int_{\Gamma_{1}} \mathbf{g} \cdot \gamma_{B}(\mathbf{u}) d s\right) \\
& +\int_{\Gamma_{0}} j_{\infty}\left(x,\left(\mathbf{u}^{0}-\gamma_{B}(\mathbf{u})\right) \otimes_{s} \boldsymbol{\nu}\right) d s
\end{aligned}
$$

and

$$
G_{j}^{* *}(\varepsilon(\mathbf{u}))=\int_{\Omega} j\left(x, \varepsilon(\mathbf{u})_{a}\right) d x+\int_{\Omega} j_{\infty}\left(x, \frac{d\left(\varepsilon(\mathbf{u})_{s}\right)}{d\left|\varepsilon(\mathbf{u})_{s}\right|}\right) d\left|\varepsilon(\mathbf{u})_{s}\right|
$$

for every $\mathbf{u} \in B D(\Omega)$ (cf. [4, (4.8), (4.12), (4.16) and (7.64)]). Here, $\left\lceil R P_{\lambda, j}^{* *}\right]$ describes the relaxed total elastic-perfectly plastic energy.

Assumption 5. There exist $\boldsymbol{\sigma}_{L} \in W^{n}(\Omega$, div $)$, where $\left\lceil P_{\lambda_{L}, j}^{*}\right\rceil\left(\lambda_{L} \boldsymbol{\sigma}_{L}\right)=$ $\sup \left\{\left\lceil P_{\lambda_{L}, j}^{*}\right\rceil(\boldsymbol{\sigma}) \mid \boldsymbol{\sigma} \in W^{n}(\Omega, \operatorname{div})\right\}<\infty$ and $0 \leq \lambda_{L}<\inf \left(P_{0, j}\right)_{A L}(\mathrm{cf}$. $(3.3))$.

Assumption 6. $\inf \left\{\left\lceil P_{\lambda_{L}, j}\right\rceil(\mathbf{u}) \mid \mathbf{u} \in B D(\Omega)\right\}=\sup \left\{\left\lceil P_{\lambda_{L}, j}^{*}\right\rceil(\boldsymbol{\sigma}) \mid \boldsymbol{\sigma} \in\right.$ $\left.W^{n}(\Omega, \operatorname{div})\right\}$, where $\lambda_{L}$ satisfies Assumption 5 (cf. [4, Theorem 14]). 
The relaxed limit analysis problem $\left(\widetilde{P}_{0, j}\right)_{A L}$ is defined by:

$$
\begin{aligned}
& \left(\widetilde{P}_{0, j}\right)_{A L} \text { find } \inf \left\{\int_{\Omega} j_{\infty}(x, \mathbf{w}) d x \mid\right. \\
& \left.\qquad \mathbf{w} \in L^{1}\left(\Omega, \mathbb{E}_{s}^{n}\right), \int_{\Omega} \boldsymbol{\sigma}_{L}: \mathbf{w} d x=1\right\} .
\end{aligned}
$$

Here, the infimum is taken over the set $\left\{\mathbf{w} \in L^{1}\left(\Omega, \mathbb{E}_{s}^{n}\right) \mid \int_{\Omega} \boldsymbol{\sigma}_{L}: \mathbf{w} d x=1\right\}$ where $\boldsymbol{\sigma}_{L}$ satisfies Assumption 5.

Due to (3.3), Assumption 5 and [4, (6.7), (6.8)], we obtain $\operatorname{div} \boldsymbol{\sigma}_{L}=-\lambda \mathbf{f}$ in $\Omega$ and $\boldsymbol{\beta}_{B}\left(\boldsymbol{\sigma}_{L}\right)=\lambda \mathbf{g}$ on $\Gamma_{1}$. If $\mathbf{w}=\boldsymbol{\varepsilon}(\mathbf{u})$ and $\mathbf{u} \in L D(\Omega) \cap C_{a}\left(\mathbf{u}^{0}\right)$, where $\mathbf{u}^{0}=\mathbf{0}$, then

$$
\begin{aligned}
\int_{\Omega} \boldsymbol{\sigma}_{L}: \mathbf{w} d x & =\int_{\Omega} \boldsymbol{\sigma}_{L}: \boldsymbol{\varepsilon}(\mathbf{u}) d x=-\int_{\Omega}\left(\operatorname{div} \boldsymbol{\sigma}_{L}\right) \cdot \mathbf{u} d x \\
& +\int_{\operatorname{Fr} \Omega} \boldsymbol{\beta}_{B}\left(\boldsymbol{\sigma}_{L}\right) \cdot \gamma_{B}(\mathbf{u}) d s=\int_{\Omega} \lambda \mathbf{f} \cdot \mathbf{u} d x+\int_{\Gamma_{1}} \lambda \mathbf{g} \cdot \boldsymbol{\gamma}_{B}(\mathbf{u}) d s
\end{aligned}
$$

(see [4, (3.9)]). Therefore, directly from (3.1) we get $\inf \left(P_{0, j}\right)_{A L} \geq \inf \left(\widetilde{P}_{0, j}\right)_{A L}$. That is, $\left(P_{0, j}\right)_{A L}$ is a stronger limit analysis problem than $\left(\widetilde{P}_{0, j}\right)_{A L}$.

Assumption 7 . For every $\widehat{r}>0$ there exists $\delta_{\widehat{r}}>0$ such that

$$
\left|j^{*}\left(x, \mathbf{w}_{1}^{*}\right)-j^{*}\left(x, \mathbf{w}_{2}^{*}\right)\right| \leq \delta_{\widehat{r}}\left\|\mathbf{w}_{1}^{*}-\mathbf{w}_{2}^{*}\right\|_{\mathbb{E}_{s}^{n}}
$$

for $d x$-a.e. $x \in \Omega$ and for all $\mathbf{w}_{1}^{*}, \mathbf{w}_{2}^{*} \in \mathcal{K}(x)$ with $\left\|\mathbf{w}_{1}^{*}\right\|_{\mathbb{E}_{s}^{n}},\left\|\mathbf{w}_{2}^{*}\right\|_{\mathbb{E}_{s}^{n}}<\widehat{r}$.

The main result of this paper is the following criterion of regularity of displacement solutions.

Theorem 1. Let $0 \leq \lambda_{L}<\lambda_{r}<\inf \left(\widetilde{P}_{0, j}\right)_{A L}$. If Assumptions 4-7 hold, then every minimum $\widetilde{\mathbf{u}} \in B D(\Omega)$ of $\left\lceil R P_{\lambda_{L}, j}^{* *}\right\rceil$ belongs to the space $L D(\Omega)$ and $\boldsymbol{\gamma}_{B}(\widetilde{\mathbf{u}})=\mathbf{0}$ on $\Gamma_{0}(c f$. (3.4) and (3.7)).

4. Coercivity of elastic-plastic energy. We now study the coercivity of $\left\lceil P_{\lambda, j}\right\rceil$ (cf. Proposition 14). Here, the elastic-perfectly plastic potential $j$ satisfies only (2.5), (2.7) and Assumptions 1-2. In this section, we always assume that Assumptions 1-3 are fulfilled, and Assumption 4 holds if it is stated explicitly. We do not assume that the set $\mathcal{K}(x)$ is bounded for each $x \in \bar{\Omega}$.

The original problem $\left(P_{\lambda, j}\right)$, defined in [4, (6.5)], is connected with the limit analysis problem $\left(P_{0, j}\right)_{A L}$ for the case when $\mathbf{u}^{0}=\mathbf{0}$ on $\Gamma_{0}$ (see Section 3). Similarly, $\left(R P_{\lambda, j}^{* *}\right)$, defined in $[4,(4.16)]$ with $\mathbf{u}^{0}=\mathbf{0}$ on $\Gamma_{0}$, is connected with the relaxed bidual limit analysis problem $\left(R P_{0, j}^{* *}\right)_{A L}$ (cf. Section 3). 
We consider the spaces

$$
\begin{aligned}
\mathbf{Y}^{1}(\bar{\Omega}) \equiv\left\{\mathbf{M} \in \mathbb{M}_{b}\left(\bar{\Omega}, \mathbb{E}_{s}^{n}\right) \mid\right. & \\
& \left.\exists \widehat{\mathbf{u}} \in B D\left(\Omega_{1}\right), \boldsymbol{\varepsilon}(\widehat{\mathbf{u}})_{\mid \bar{\Omega}}=\mathbf{M}, \widehat{\mathbf{u}}_{\mid \Omega_{1}-\bar{\Omega}}=\mathbf{0}\right\} \\
C\left(\bar{\Omega}, \mathbb{E}_{s}^{n}\right) \cap W^{n}(\Omega, \operatorname{div}) & \equiv\left\{\boldsymbol{\sigma} \in C\left(\bar{\Omega}, \mathbb{E}_{s}^{n}\right) \mid \boldsymbol{\sigma}_{\mid \Omega} \in W^{n}(\Omega, \operatorname{div})\right\}
\end{aligned}
$$

Let $\boldsymbol{\varepsilon}(\mathbf{u})_{\mid \bar{\Omega}}=\mathbf{M}$, where $\mathbf{u} \in B D\left(\Omega_{1}\right)$ and $\mathbf{u}_{\mid \Omega_{1}-\bar{\Omega}}=\mathbf{0}$. Moreover, let $\boldsymbol{\sigma}_{1} \in$ $W^{n}\left(\Omega_{1}\right.$, div) where $\boldsymbol{\sigma}_{1 \mid \Omega}=\boldsymbol{\sigma}$ (see [4, Remark 1]). Then we define

$$
\langle\mathbf{M}, \boldsymbol{\sigma}\rangle_{\mathbf{Y}^{1} \times W^{n}(\Omega, \operatorname{div})}=\int_{\bar{\Omega}} \boldsymbol{\sigma}_{1}: \boldsymbol{\varepsilon}(\mathbf{u})=\int_{\Omega} \boldsymbol{\sigma}: \boldsymbol{\varepsilon}(\mathbf{u})_{\mid \Omega}-\int_{\text {Fr } \Omega} \boldsymbol{\beta}_{B}(\boldsymbol{\sigma}) \cdot \gamma_{B}^{I}(\mathbf{u}) d s
$$

(cf. [3, Lemma 5 and Remark 1], [4, (3.8), (5.4) and (5.5)]).

REMARK 1. We should consider duality between $\mathbf{Y}^{1}(\bar{\Omega})$ and $\left[C\left(\bar{\Omega}, \mathbb{E}_{s}^{n}\right) \cap\right.$ $\left.W^{n}(\Omega, \operatorname{div})\right] /\left\{\boldsymbol{\sigma} \in C\left(\bar{\Omega}, \mathbb{E}_{s}^{n}\right) \mid \operatorname{div} \boldsymbol{\sigma}=\mathbf{0}\right.$ in $\left.\Omega\right\}$ or another quotient space. To simplify the proofs, the definitions (4.1)-(4.3) are considered. We do not obtain a contradiction, since we do not use the Hausdorff property of $\sigma\left(W^{n}(\Omega, \operatorname{div}), \mathbf{Y}^{1}(\bar{\Omega})\right)$ and $\sigma\left(C\left(\bar{\Omega}, \mathbb{E}_{s}^{n}\right) \cap W^{n}(\Omega, \operatorname{div}), \mathbf{Y}^{1}(\bar{\Omega})\right)$.

The space $B D(\Omega)$ is isomorphic to $\mathcal{A} \equiv\left\{\mathbf{u} \in B D\left(\Omega_{1}\right) \mid \mathbf{u}_{\mid \Omega_{1}-\bar{\Omega}}=\mathbf{0}\right\}$. Moreover, $\mathcal{A}$ is isomorphic to $\mathbf{Y}^{1}(\bar{\Omega})$ via $\mathcal{A} \ni \mathbf{u} \mapsto \varepsilon(\mathbf{u})_{\mid \bar{\Omega}} \in \mathbf{Y}^{1}(\bar{\Omega})$. The Banach spaces $\left[B D(\Omega),\|\cdot\|_{B D}\right]$ and $\left[\mathbf{Y}^{1}(\bar{\Omega}),\|\cdot\|_{\mathbb{M}_{b}(\bar{\Omega})}\right]$ are isomorphic (cf. [2, Proposition 4.24]). Each closed ball cl $\mathrm{l}_{\|\cdot\|}\left(B_{\mathbf{Y}^{1}}(0, r)\right)\left(\right.$ in $\left.\mathbf{Y}^{1}\right)$ is compact in the topology $\sigma\left(\mathbf{Y}^{1}(\bar{\Omega}) ; C\left(\bar{\Omega}, \mathbb{E}_{s}^{n}\right) \cap W^{n}(\Omega\right.$, div $\left.)\right)$, where $\operatorname{cl}_{\|\cdot\|}$ denotes the closure in the norm of $B D(\Omega)$ (see [2, Proposition 4.23]). The space $\left[\mathrm{cl}_{\|\cdot\|_{B D}}\left(B_{B D}(0, r)\right)\right.$, weak* $B D(\Omega)$ topology $]$ is homeomorphic to the space $\left[\operatorname{cl}_{\|\cdot\|_{B D}}\left(B_{B D}(0, r)\right), \sigma\left(\mathbf{Y}^{1}(\bar{\Omega}) ; C\left(\bar{\Omega}, \mathbb{E}_{s}^{n}\right) \cap W^{n}(\Omega\right.\right.$, div $\left.\left.)\right)\right]$ for every $r>0$ (cf. [2, Proposition 4.25]).

We say that a net $\left\{\mathbf{M}_{\delta}\right\}_{\delta \in D} \subset \mathbf{Y}^{1}(\bar{\Omega})$ is convergent to $\mathbf{M}_{0} \in \mathbf{Y}^{1}(\bar{\Omega})$ in the topology $\sigma\left(\mathbf{Y}^{1}(\bar{\Omega}), W^{n}(\Omega, \operatorname{div})\right)$ if

$$
\left\langle\left(\mathbf{M}_{\delta}-\mathbf{M}_{0}\right), \boldsymbol{\sigma}\right\rangle_{\mathbf{Y}^{1} \times W^{n}(\Omega, \operatorname{div})} \rightarrow 0 \quad \forall \boldsymbol{\sigma} \in W^{n}(\Omega, \operatorname{div}) .
$$

Proposition 2. Each closed ball $\mathrm{cl}_{\|\cdot\|_{\mathbb{M}_{b}}}\left(B_{\mathbf{Y}^{1}}(0, r)\right)\left(\right.$ in $\left.\mathbf{Y}^{1}(\bar{\Omega})\right)$ is compact in $\sigma\left(\mathbf{Y}^{1}(\bar{\Omega}), W^{n}(\Omega\right.$, div $\left.)\right)$. If $n=1$ then $L^{n /(n-1)}(\Omega)^{n}$ shoud be replaced by $L^{\infty}(\Omega)$ in the proof below.

Proof. Step 1. Let $\left\{\varepsilon\left(\mathbf{u}_{\delta}\right)_{\mid \bar{\Omega}}\right\}_{\delta \in D} \subset \mathbf{Y}^{1}(\bar{\Omega})$ be bounded in the norm $\|\cdot\|_{\mathbb{M}_{b}(\bar{\Omega})}$. Then $\left\{\mathbf{u}_{\delta \mid \Omega}\right\}_{\delta \in D} \subset B D(\Omega)$ is bounded in $\|\cdot\|_{B D}$. There exists a continuous injection of $B D(\Omega)$ into $L^{n /(n-1)}(\Omega)^{n}$ (see [16, Chapter 2, Theorem 2.2]). Thus $\left\{\mathbf{u}_{\delta \mid \Omega}\right\}_{\delta \in D}$ is a bounded net in $L^{n /(n-1)}$. Therefore, there exist a finer net $\left\{\mathbf{u}_{\delta_{\alpha}}\right\}_{\alpha \in A} \subset\left\{\mathbf{u}_{\delta}\right\}_{\delta \in D}$ and a function $\mathbf{u}_{1} \in L^{n /(n-1)}(\Omega)^{n}$ such that 


$$
\left\langle\varepsilon\left(\mathbf{u}_{\delta_{\alpha}}\right), \boldsymbol{\sigma}\right\rangle_{\mathbf{Y}^{1} \times W^{n}(\Omega, \operatorname{div})}=-\int_{\Omega}(\operatorname{div} \boldsymbol{\sigma}) \cdot \mathbf{u}_{\delta_{\alpha}} d x \rightarrow-\int_{\Omega}(\operatorname{div} \boldsymbol{\sigma}) \cdot \mathbf{u}_{1} d x
$$

for every $\boldsymbol{\sigma} \in W^{n}(\Omega, \operatorname{div})$, since $\operatorname{div} \boldsymbol{\sigma} \in L^{n}(\Omega)^{n}$. Moreover, there is a finer net $\left\{\mathbf{u}_{\delta_{\alpha_{\beta}}}\right\}$ and a measure $\boldsymbol{\mu}_{1} \in \mathbb{M}_{b}\left(\Omega, \mathbb{E}_{s}^{n}\right)$ such that $\int_{\Omega} \boldsymbol{\varphi}: \varepsilon\left(\mathbf{u}_{\delta_{\alpha_{\beta}}}\right) \rightarrow \int_{\Omega} \boldsymbol{\varphi}: \boldsymbol{\mu}_{1}$ for every $\varphi \in C_{c}^{1}\left(\Omega, \mathbb{E}_{s}^{n}\right)$. The symmetric distributional derivative $\varepsilon\left(\mathbf{u}_{1}\right)$ of $\mathbf{u}_{1}$ is equal to $\boldsymbol{\mu}_{1}$, since $C_{c}^{1}\left(\Omega_{1}, \mathbb{E}_{s}^{n}\right) \subset W^{n}\left(\Omega_{1}\right.$, div $)$. Then $\mathbf{u}_{1} \in B D(\Omega)$ and $\varepsilon\left(\mathbf{u}_{\delta_{\alpha_{\beta}}}\right)_{\mid \bar{\Omega}}$ converges to $\varepsilon\left(\widetilde{\mathbf{u}}_{1}\right)_{\mid \bar{\Omega}}$ in $\sigma\left(\mathbf{Y}^{1}(\bar{\Omega}), W^{n}(\Omega\right.$, div $\left.)\right)$, where $\widetilde{\mathbf{u}}_{1} \in$ $B D\left(\Omega_{1}\right), \widetilde{\mathbf{u}}_{1 \mid \Omega}=\mathbf{u}_{1}$ in $\Omega$ and $\widetilde{\mathbf{u}}_{1 \mid \Omega_{1}-\bar{\Omega}}=\mathbf{0}$ in $\Omega_{1}-\bar{\Omega}$.

Step 2. The net $\left\{\varepsilon\left(\mathbf{u}_{\delta}\right)_{\mid \bar{\Omega}}\right\}_{\delta \in D} \subset \mathbf{Y}^{1}$ is contained in $\mathrm{cl}_{\|\cdot\|_{\mathbb{M}_{b}}}\left(B_{\mathbf{Y}^{1}}(0, r)\right)$. Then

$$
\begin{array}{r}
\left\|\varepsilon\left(\widetilde{\mathbf{u}}_{1}\right)_{\mid \bar{\Omega}}\right\|_{\mathbb{M}_{b}} \leq \sup _{\boldsymbol{\sigma} \in C_{0}^{1}}\left\{\limsup _{\delta}\left\langle\boldsymbol{\varepsilon}\left(\mathbf{u}_{\delta}\right), \boldsymbol{\sigma}\right\rangle_{\mathbf{Y}^{1} \times W^{n}\left(\Omega_{1}, \text { div }\right)} \mid\right. \\
\left.\|\boldsymbol{\sigma}(x)\|_{\mathbb{E}_{s}^{n}} \leq 1, \forall x \in \Omega_{1}\right\} \leq r .
\end{array}
$$

TheOREM 3. The topologies $\sigma\left(\mathbf{Y}^{1}(\bar{\Omega}) ; C\left(\bar{\Omega}, \mathbb{E}_{s}^{n}\right) \cap W^{n}(\Omega\right.$, div $\left.)\right)$ and $\sigma\left(\mathbf{Y}^{1}(\bar{\Omega}), W^{n}(\Omega, \operatorname{div})\right)$ are equivalent on each closed ball $\mathrm{cl}_{\|\cdot\|_{\mathbb{M}_{b}}}\left(B_{\mathbf{Y}^{1}(\bar{\Omega})}(0, r)\right)$.

Proof. The topology $\sigma\left(\mathbf{Y}^{1}(\bar{\Omega}) ; C\left(\bar{\Omega}, \mathbb{E}_{s}^{n}\right) \cap W^{n}(\Omega, \operatorname{div})\right)$ is weaker than $\sigma\left(\mathbf{Y}^{1}(\bar{\Omega}), W^{n}(\Omega, \operatorname{div})\right)$. Moreover, $\sigma\left(\mathbf{Y}^{1}(\bar{\Omega}) ; C\left(\bar{\Omega}, \mathbb{E}_{s}^{n}\right) \cap W^{n}(\Omega, \operatorname{div})\right)$ is a Hausdorff topology and $\left[\mathrm{cl}_{\|\cdot\|_{\mathbb{M}_{b}}}\left(B_{\mathbf{Y}^{1}}(0, r)\right), \sigma\left(\mathbf{Y}^{1}(\bar{\Omega}), W^{n}(\Omega, \operatorname{div})\right)\right]$ is a compact topological space. Among all Hausdorff topologies, compact topologies are minimal (see [9, Corollary 3.1.14]).

Lemma 4. The functional $L:\left[B D(\Omega),\|\cdot\|_{B D}\right] \rightarrow \mathbb{R}$ is continuous (see $(2.10))$.

Proof. The trace $\gamma_{B}:\left[B D(\Omega),\|\cdot\|_{B D}\right] \rightarrow\left[L^{1}(\operatorname{Fr} \Omega)^{n},\|\cdot\|_{L^{1}}\right]$ is continuous (cf. [16, Chapter 2, Theorem 1.1]). Moreover, $B D(\Omega) \ni \mathbf{u} \mapsto \int_{\Omega} \mathbf{f} \cdot \mathbf{u} d x \in$ $\mathbb{R}$ is continuous in $\|\cdot\|_{B D}$ (see [16, Chapter 2, Theorem 1.2]).

Lemma 5. If $d s\left(\Gamma_{0}\right)>0$, then for every $\mathbf{g} \in L^{\infty}\left(\Gamma_{1}\right)^{n}, \mathbf{f} \in L^{n}(\Omega)^{n}$ there exists $\widehat{\boldsymbol{\sigma}} \in W^{n}(\Omega, \operatorname{div})$ with $\operatorname{div} \widehat{\boldsymbol{\sigma}}=-\mathbf{f}$ in $\Omega$ and $\boldsymbol{\beta}_{B}(\widehat{\boldsymbol{\sigma}})=\mathbf{g}$ on $\Gamma_{1}$.

If $d s\left(\Gamma_{0}\right)=0$, then for every $\mathbf{g} \in L^{\infty}\left(\Gamma_{1}\right)^{n}, \mathbf{f} \in L^{n}(\Omega)^{n}$ (such that for every $\mathbf{u} \in L D(\Omega)$ and $\overline{\mathbf{u}} \in L D(\Omega)$ with $\varepsilon(\overline{\mathbf{u}})=\mathbf{0}$ we have $L(\mathbf{u})=L(\mathbf{u}+\overline{\mathbf{u}}))$ there exists $\widehat{\boldsymbol{\sigma}}_{0} \in W^{n}(\Omega$, div $)$ with $\operatorname{div} \widehat{\boldsymbol{\sigma}}_{0}=-\mathbf{f}$ in $\Omega$ and $\boldsymbol{\beta}_{B}\left(\widehat{\boldsymbol{\sigma}}_{0}\right)=\mathbf{g}$ on $\operatorname{Fr} \Omega$.

Proof. Step 1. Let $\widehat{j}_{m}: \Omega \times \mathbb{E}_{s}^{n} \rightarrow \mathbb{R}$ be defined by

$$
\widehat{j}_{m}(x, \mathbf{w}) \equiv \sup \left\{\mathbf{w}: \mathbf{w}^{*} \mid \mathbf{w}^{*} \in \mathbb{E}_{s}^{n},\left\|\mathbf{w}^{*}\right\|_{\mathbb{E}_{s}^{n}} \leq m\right\} .
$$

Then $\widehat{j}_{m}$ is a normal integrand for every $m \in \mathbb{N}$. We have $\widehat{j}_{m}(x, \mathbf{w}) \geq$ $m c_{n}\|\mathbf{w}\|_{\mathbb{E}_{s}^{n}}$ for every $m \in \mathbb{N}, x \in \Omega$ and $\mathbf{w} \in \mathbb{E}_{s}^{n}$, where $c_{n}$ depends only on the dimension of the space $\mathbb{E}_{s}^{n}$ (cf. definition of the norm $\|\cdot\|_{\mathbb{E}_{s}^{n}}$ ). 
Step 2. Let $d s\left(\Gamma_{0}\right)>0$. For every $\mathbf{u} \in L D(\Omega)$ such that $\gamma_{B}(\mathbf{u})=\mathbf{0}$ on $\Gamma_{0}$, there exists $c^{0}>0$ such that $\int_{\Omega}\|\varepsilon(\mathbf{u})\|_{\mathbb{E}_{s}^{n}} d x \geq c^{0}\|\mathbf{u}\|_{L D(\Omega)}$. Then

(4.8) $\inf _{\mathbf{u} \in L D(\Omega)}\left\{\int_{\Omega} \widehat{j}_{m}(x, \varepsilon(\mathbf{u})) d x \mid \gamma_{B}(\mathbf{u})=\mathbf{0}\right.$ on $\left.\Gamma_{0},\|\mathbf{u}\|_{L D}=1\right\} \geq m c_{n} c^{0}$.

We take $m_{0} \in \mathbb{N}$ such that $\sup \left\{|L(u)| \mid \mathbf{u} \in L D(\Omega),\|\mathbf{u}\|_{L D(\Omega)}=1\right\}<$ $m_{0} c_{n} c^{0}$; then $\inf \left\{\left\lceil P_{1, \widehat{j}_{m_{0}}}\right\rceil(\mathbf{u}) \mid \mathbf{u} \in L D(\Omega)\right\}>-\infty$ (cf. (2.9)). Therefore, $\left(P_{1, \widehat{j}_{m_{0}}}^{*}\right)$ has a solution (see [4, Theorem 14 and Lemma 13]).

Step 3. Let $d s\left(\Gamma_{0}\right)=0$. Then, for every $\mathbf{u} \in L D(\Omega)$, there exists $c^{0}>0$ such that

$$
\int_{\Omega}\|\varepsilon(\mathbf{u})\|_{\mathbb{E}_{s}^{n}} d x \geq c^{0} \inf \left\{\|\mathbf{u}+\overline{\mathbf{u}}\|_{L D(\Omega)} \mid \overline{\mathbf{u}} \in L D(\Omega), \boldsymbol{\varepsilon}(\overline{\mathbf{u}})=\mathbf{0}\right\} .
$$

We have

$$
\begin{aligned}
\inf _{\mathbf{u}}\left\{\int_{\Omega} \widehat{j}_{m}(x, \boldsymbol{\varepsilon}(\mathbf{u})) d x \mid \mathbf{u} \in L D(\Omega)\right. \text { and } \\
\left.\quad \inf _{\overline{\mathbf{u}}}\left\{\|\mathbf{u}+\overline{\mathbf{u}}\|_{L D(\Omega)} \mid \overline{\mathbf{u}} \in L D(\Omega), \boldsymbol{\varepsilon}(\overline{\mathbf{u}})=\mathbf{0}\right\}=1\right\} \geq m c_{n} c^{0} .
\end{aligned}
$$

This yields the second part of this lemma (cf. Step 2).

We define a subspace $G L D(\Omega)$ of $L^{1}\left(\Omega, \mathbb{E}_{s}^{n}\right) \times L^{1}(\operatorname{Fr} \Omega)^{n}$ by

$$
G L D(\Omega) \equiv\left\{(\mathbf{w}, \mathbf{z}) \mid \exists \widetilde{\mathbf{u}} \in L D(\Omega),(\mathbf{w}, \mathbf{z})=\left(\varepsilon(\widetilde{\mathbf{u}}), \boldsymbol{\gamma}_{B}(\widetilde{\mathbf{u}})\right)\right\} .
$$

Let $\widetilde{\Theta}_{\lambda}: L^{1}\left(\Omega, \mathbb{E}_{s}^{n}\right) \times L^{1}(\operatorname{Fr} \Omega)^{n} \rightarrow \mathbb{R} \cup\{+\infty\}$ be given by

$$
\begin{aligned}
\widetilde{\Theta}_{\lambda}\left(\mathbf{w}, \gamma_{B}(\mathbf{u})\right)= & -\lambda \int_{\Gamma_{1}} \mathbf{g} \cdot \boldsymbol{\gamma}_{B}(\mathbf{u}) d s+\int_{\Omega} j_{\infty}(x, \mathbf{w}) d x \\
& +\int_{\Gamma_{0}} I_{\left\{\gamma_{B}(\mathbf{u}) \otimes_{s} \boldsymbol{\nu}=\mathbf{0}\right\}}\left(-\gamma_{B}(\mathbf{u}) \otimes_{s} \boldsymbol{\nu}\right) d s
\end{aligned}
$$

for $\mathbf{w} \in L^{1}\left(\Omega, \mathbb{E}_{s}^{n}\right)$ and $\mathbf{u} \in L D(\Omega)$ (note that $\left.\boldsymbol{\gamma}_{B}(L D(\Omega))=L^{1}(\operatorname{Fr} \Omega)^{n}\right)$. The restriction of $\widetilde{\Theta}_{\lambda}$ to $G L D(\Omega)$ is equal to $\Theta_{\lambda}$. Let $W^{n}(\Omega, \operatorname{div})$ and $L^{1}\left(\Omega, \mathbb{E}_{s}^{n}\right) \times L^{1}(\operatorname{Fr} \Omega)^{n}$ be vector spaces placed in duality by the bilinear pairing

$$
\langle\boldsymbol{\sigma},(\mathbf{w}, \mathbf{p})\rangle_{2}=\int_{\Omega} \boldsymbol{\sigma}: \mathbf{w} d x-\int_{\operatorname{Fr} \Omega} \boldsymbol{\beta}_{B}(\boldsymbol{\sigma}) \cdot \mathbf{p} d s
$$

for $\boldsymbol{\sigma} \in W^{n}(\Omega, \operatorname{div}), \mathbf{w} \in L^{1}\left(\Omega, \mathbb{E}_{s}^{n}\right)$ and $\mathbf{p} \in L^{1}(\operatorname{Fr} \Omega)^{n}$. In view of the duality we obtain

$$
\begin{array}{r}
\widetilde{\Theta}_{\lambda}^{\#}(\boldsymbol{\sigma}) \equiv \sup \left\{\left\langle\boldsymbol{\sigma},\left(\mathbf{w}, \boldsymbol{\gamma}_{B}(\mathbf{u})\right)\right\rangle_{2}-\widetilde{\Theta}_{\lambda}\left(\mathbf{w}, \boldsymbol{\gamma}_{B}(\mathbf{u})\right) \mid\right. \\
\left.\left(\mathbf{w}, \boldsymbol{\gamma}_{B}(\mathbf{u})\right) \in L^{1} \times L^{1}\right\}
\end{array}
$$


for every $\boldsymbol{\sigma} \in W^{n}(\Omega, \operatorname{div})$, and

$$
\widetilde{\Theta}_{\lambda}^{\# \#}\left(\mathbf{w}, \gamma_{B}(\mathbf{u})\right)=\sup \left\{\left\langle\boldsymbol{\sigma},\left(\mathbf{w}, \gamma_{B}(\mathbf{u})\right)\right\rangle_{2}-\widetilde{\Theta}_{\lambda}^{\#}(\boldsymbol{\sigma}) \mid \boldsymbol{\sigma} \in W^{n}(\Omega, \operatorname{div})\right\}
$$
for every $\mathbf{w} \in L^{1}\left(\Omega, \mathbb{E}_{s}^{n}\right)$ and $\mathbf{u} \in L D(\Omega)$. Moreover, due to the duality between $W^{n}\left(\Omega\right.$, div) and $G L D(\Omega)$, we define $\Theta_{\lambda}^{\#}$ and $\Theta_{\lambda}^{\# \#}: G L D(\Omega) \rightarrow$ $\mathbb{R} \cup\{+\infty\}$ by

$$
(4.16) \Theta_{\lambda}^{\#}(\boldsymbol{\sigma}) \equiv \sup _{\left(\boldsymbol{\varepsilon}(\mathbf{u}), \boldsymbol{\gamma}_{B}(\mathbf{u})\right) \in G L D(\Omega)}\left\{\left\langle\boldsymbol{\sigma},\left(\boldsymbol{\varepsilon}(\mathbf{u}), \boldsymbol{\gamma}_{B}(\mathbf{u})\right)\right\rangle_{2}-\Theta_{\lambda}\left(\varepsilon(\mathbf{u}), \boldsymbol{\gamma}_{B}(\mathbf{u})\right)\right\}
$$

for $\boldsymbol{\sigma} \in W^{n}(\Omega, \operatorname{div})$, and

$$
\begin{aligned}
& \Theta_{\lambda}^{\# \#}\left(\boldsymbol{\varepsilon}(\mathbf{u}), \gamma_{B}(\mathbf{u})\right) \\
& \quad=\sup \left\{\left\langle\boldsymbol{\sigma},\left(\boldsymbol{\varepsilon}(\mathbf{u}), \gamma_{B}(\mathbf{u})\right)\right\rangle_{2}-\Theta_{\lambda}^{\#}(\boldsymbol{\sigma}) \mid \boldsymbol{\sigma} \in W^{n}(\Omega, \operatorname{div})\right\} .
\end{aligned}
$$

Similarly to [2, (4.62)], we obtain

Proposition 6. We have

$$
\widetilde{\Theta}_{\lambda}^{\#}(\boldsymbol{\sigma})=\int_{\Omega} j_{\infty}^{*}(x, \boldsymbol{\sigma}) d x+\int_{\Gamma_{1}} I_{\left\{\boldsymbol{\sigma} \mid \boldsymbol{\beta}_{B}(\boldsymbol{\sigma})=\lambda \mathbf{g}\right\}}(\boldsymbol{\sigma}) d s
$$

for every $\boldsymbol{\sigma} \in W^{n}(\Omega, \operatorname{div})$. If $\lambda_{r}$ satisfies Assumption 4, then

$$
\begin{aligned}
\widetilde{\Theta}_{\lambda_{r}}^{\# \#}\left(\mathbf{w}, \gamma_{B}(\mathbf{u})\right)= & -\lambda_{r} \int_{\Gamma_{1}} \mathbf{g} \cdot \gamma_{B}(\mathbf{u}) d s+\int_{\Omega} j_{\infty}(x, \mathbf{w}) d x \\
& +\int_{\Gamma_{0}} j_{\infty}\left(x,-\gamma_{B}(\mathbf{u}) \otimes_{s} \boldsymbol{\nu}\right) d s
\end{aligned}
$$

for every $\mathbf{w} \in L^{1}\left(\Omega, \mathbb{E}_{s}^{n}\right)$ and $\mathbf{u} \in L D(\Omega)$ (see [3, Proposition 7]).

Lemma 7 (see [3, Lemma 6]). For every $\boldsymbol{\sigma} \in W^{n}(\Omega, \operatorname{div})$ we have $\widetilde{\Theta}_{\lambda}^{\#}(\boldsymbol{\sigma})$ $\geq \Theta_{\lambda}^{\#}(\boldsymbol{\sigma})$. Moreover, $\widetilde{\Theta}_{\lambda}^{\# \#}\left(\varepsilon(\mathbf{u}), \gamma_{B}(\mathbf{u})\right) \leq \Theta_{\lambda}^{\# \#}\left(\varepsilon(\mathbf{u}), \gamma_{B}(\mathbf{u})\right)$ for every $\left(\varepsilon(\mathbf{u}), \gamma_{B}(\mathbf{u})\right) \in G L D(\Omega)$.

LEMma 8 (cf. [3, Lemma 8]). Let $\lambda_{r}$ satisfy Assumption 4. For every $\mathbf{u} \in L D(\Omega)$ such that $\boldsymbol{\gamma}_{B}(\mathbf{u})=\mathbf{0}$ on $\Gamma_{0}$, we have

$$
\widetilde{\Theta}_{\lambda_{r}}^{\# \#}\left(\varepsilon(\mathbf{u}), \gamma_{B}(\mathbf{u})\right)=\Theta_{\lambda_{r}}^{\# \#}\left(\varepsilon(\mathbf{u}), \gamma_{B}(\mathbf{u})\right)=\Theta_{\lambda_{r}}\left(\varepsilon(\mathbf{u}), \gamma_{B}(\mathbf{u})\right)
$$

LEMma 9 (see [3, Lemma 9]). For every $\boldsymbol{\sigma} \in W^{n}(\Omega, \operatorname{div})$ and every $\boldsymbol{\sigma}_{s} \in W^{n}(\Omega, \operatorname{div})$ such that $\operatorname{div} \boldsymbol{\sigma}_{s}=\mathbf{0}$, we have $\Theta_{\lambda}^{\#}(\boldsymbol{\sigma})=\Theta_{\lambda}^{\#}\left(\boldsymbol{\sigma}+\boldsymbol{\sigma}_{s}\right)$.

We say that the net $\left\{\boldsymbol{\sigma}_{\kappa}\right\}_{\kappa \in K} \subset W^{n}\left(\Omega\right.$, div) converges to $\boldsymbol{\sigma}_{0} \in W^{n}(\Omega$, div $)$ in

$$
\sigma\left(W^{n}(\Omega, \operatorname{div}) ; L^{1}\left(\Omega, \mathbb{E}_{s}^{n}\right) \times\left\{\boldsymbol{\varphi} \in \mathbf{Y}^{1}(\bar{\Omega})_{\mid \operatorname{Fr} \Omega} \mid \boldsymbol{\varphi}_{\mid \Gamma_{0}}=\mathbf{0}\right\}\right),
$$

if $\left\langle\boldsymbol{\sigma}_{\kappa},(\mathbf{w}, \mathbf{p})\right\rangle_{2} \rightarrow\left\langle\boldsymbol{\sigma}_{0},(\mathbf{w}, \mathbf{p})\right\rangle_{2}$ for every $(\mathbf{w}, \mathbf{p}) \in L^{1}\left(\Omega, \mathbb{E}_{s}^{n}\right) \times L^{1}(\operatorname{Fr} \Omega)^{n}$ such that $\mathbf{p}_{\mid \Gamma_{0}}=\mathbf{0}$ (note that $\mathbf{Y}^{1}(\bar{\Omega})_{\mid \operatorname{Fr} \Omega}=\gamma_{B}(L D(\Omega)) \otimes_{s} \boldsymbol{\nu}$ ). 
Lemma 10. Let $\widehat{f}: W^{n}(\Omega, \operatorname{div}) \rightarrow \mathbb{R}$ be a linear functional, continuous in the topology (4.21), such that $\widehat{f}\left(\boldsymbol{\sigma}_{s}\right)=0$ for every $\boldsymbol{\sigma}_{s} \in W^{n}(\Omega$, div) with $\operatorname{div} \boldsymbol{\sigma}_{s}=\mathbf{0}$ in $\Omega$. Then there exists $\widetilde{\mathbf{u}} \in L D(\Omega)$ such that for every $\boldsymbol{\sigma} \in W^{n}(\Omega$, div $)$, we have $\widehat{f}(\boldsymbol{\sigma})=\left\langle\boldsymbol{\sigma},\left(\varepsilon(\widetilde{\mathbf{u}}), \boldsymbol{\gamma}_{B}(\widetilde{\mathbf{u}})\right)\right\rangle_{2}$ and $\boldsymbol{\gamma}_{B}(\widetilde{\mathbf{u}})=\mathbf{0}$ on $\Gamma_{0}$ (cf. (4.13)).

Proof. It is a modification of the proof of [3, Lemma 10]. By [7, Theorem V.3.9] there exist $\mathbf{m} \in L^{1}\left(\Omega, \mathbb{E}_{s}^{n}\right)$ and $\widehat{\mathbf{u}} \in B D(\Omega)$ such that $\gamma_{B}(\widehat{\mathbf{u}})=\mathbf{0}$ on $\Gamma_{0}$ and $\widehat{f}(\boldsymbol{\sigma})=\left\langle\boldsymbol{\sigma},\left(\mathbf{m}, \boldsymbol{\gamma}_{B}(\widehat{\mathbf{u}})\right)\right\rangle_{2}$ for all $\boldsymbol{\sigma} \in W^{n}(\Omega$, div $)$. For every $\boldsymbol{\sigma}_{1} \in C\left(\bar{\Omega}_{1}, \mathbb{E}_{s}^{n}\right) \cap W^{n}\left(\Omega_{1}\right.$, div $)$ such that $\operatorname{div} \boldsymbol{\sigma}_{1}=\mathbf{0}$ in $\Omega_{1}$, we have $\left\langle\boldsymbol{\sigma}_{1 \mid \Omega}\right.$, $\left.\left(\mathbf{m}, \gamma_{B}(\widehat{\mathbf{u}})\right)\right\rangle_{2}=0\left(\right.$ since $\left.\boldsymbol{\sigma}_{1 \mid \Omega} \in W^{n}(\Omega, \operatorname{div})\right)$. Then by [16, Chapter 2, Proposition 1.1, Theorem 1.3], [4, (5.5)] and [13], there exists $\widetilde{\mathbf{u}} \in L D(\Omega)$ such that the conclusion of this lemma holds.

Let $Q: W^{n}(\Omega, \operatorname{div}) \rightarrow \mathbb{R} \cup\{+\infty\}$ be defined by

$$
Q(\boldsymbol{\sigma})=\inf _{\boldsymbol{\sigma}_{s}}\left\{\widetilde{\Theta}_{\lambda_{r}}^{\#}\left(\boldsymbol{\sigma}+\boldsymbol{\sigma}_{s}\right) \mid \boldsymbol{\sigma}_{s} \in W^{n}(\Omega, \operatorname{div}) \text { and } \operatorname{div} \boldsymbol{\sigma}_{s}=\mathbf{0}\right\} .
$$

Proposition 11. Let $\lambda_{r}$ satisfy Assumption 4. For every $\boldsymbol{\sigma} \in W^{n}(\Omega, \operatorname{div})$ we have

$$
\Theta_{\lambda_{r}}^{\#}(\boldsymbol{\sigma})=\mathrm{cl}_{(4.21)} Q(\boldsymbol{\sigma}),
$$

where $\operatorname{cl}_{(4.21)} Q$ denotes the largest minorant which is less than $Q$ and is l.s.c. in the topology (4.21) (i.e., $\operatorname{cl}_{(4.21)} Q$ is the l.s.c. regularization of $Q$ in (4.21)).

Proof. We prove the proposition in the same way as [3, Proposition 11], with $C_{\operatorname{div}}\left(\bar{\Omega}, \mathbb{E}_{s}^{n}\right)$ and the topology $[3,(4.12)]$ replaced with $W^{n}(\Omega, \operatorname{div})$ and (4.21).

Proposition 12 (see [4, Proposition 19]). Let $A_{\widetilde{k}} \equiv\left\{\boldsymbol{\sigma} \in W^{n}(\Omega, \operatorname{div}) \mid\right.$ $\left.\|\operatorname{div} \boldsymbol{\sigma}\|_{L^{n}} \leq \widetilde{k}\right\}$ and let $\lambda_{r}$ satisfy Assumption 4. For all $\widehat{\boldsymbol{\sigma}} \in W^{n}(\Omega, \operatorname{div})$ and all $\widetilde{k}>\|\operatorname{div} \widehat{\boldsymbol{\sigma}}\|_{L^{n}}$ we have

$$
\Theta_{\lambda_{r}}^{\#}(\widehat{\boldsymbol{\sigma}})=\operatorname{cl}_{A_{\widetilde{k}}} Q(\widehat{\boldsymbol{\sigma}}),
$$

where $\operatorname{cl}_{A_{\tilde{k}}} Q$ is the l.s.c. regularization of the function $\boldsymbol{\sigma} \mapsto Q(\boldsymbol{\sigma})+I_{A_{\tilde{k}}}(\boldsymbol{\sigma})$ in the topology (4.21) and $I_{A_{\tilde{k}}}(\cdot)$ is the indicator function of $A_{\widetilde{k}}$.

Proof. We argue as for [3, Proposition 13], replacing $C_{\mathrm{div}}\left(\bar{\Omega}, \mathbb{E}_{s}^{n}\right)$ with $W^{n}(\Omega$, div). In the proof, we use the topology (4.35) of [3].

If $d s\left(\Gamma_{0}\right)=0$ and $\infty>\inf \left(P_{0, j}\right)_{A L}>0$ then for every $\mathbf{u}, \overline{\mathbf{u}} \in L D(\Omega)$ such that $\varepsilon(\overline{\mathbf{u}})=\mathbf{0}$ in $\Omega$, we have $L(\mathbf{u})=L(\mathbf{u}+\overline{\mathbf{u}})($ cf. (2.10)).

Proposition 13. Let $0<\lambda_{r} \leq \inf \left(P_{0, j}\right)_{A L}$, where $\lambda_{r}$ satisfies Assumption 4. Moreover, let $\left\lceil P_{\lambda_{r}, j_{\infty}}^{\mathbf{f}_{s}}\right\rceil^{*}$ be equal to $\left\lceil P_{\lambda_{r}, j_{\infty}}^{*}\right\rceil$, where $\mathbf{f}$ is replaced by $\mathbf{f}_{s}$ (cf. (2.10), (3.3), [4. (6.6)]). Then there exists a sequence $\left\{\boldsymbol{\sigma}_{m}\right\}_{m \in \mathbb{N}} \subset$ 
$W^{n}(\Omega$, div $)$ such that $\boldsymbol{\beta}_{B}\left(\boldsymbol{\sigma}_{m}\right)=\lambda_{r} \mathbf{g}$ on $\Gamma_{1}$ for every $m \in \mathbb{N}$ and $\| \lambda_{r} \mathbf{f}+$ $\operatorname{div} \boldsymbol{\sigma}_{m} \|_{L^{n}} \rightarrow 0$. Here, $\boldsymbol{\sigma}_{m}(x) \in \mathcal{K}(x)$ for $d x$-a.e. $x \in \Omega$ and every $m \in \mathbb{N}$. Moreover, if $\mathbf{u}^{0}=\mathbf{0}$ on $\Gamma_{0}$, then $\left\lceil P_{\lambda_{r}, j_{\infty}}^{-\operatorname{div} \boldsymbol{\sigma}_{m}}\right\rceil^{*}$ has a maximum in $W^{n}(\Omega, \operatorname{div})$, for each $m \in \mathbb{N}$.

Proof. Let $\mathbf{u}^{0}=\mathbf{0}$ on $\Gamma_{0}$. Then $0 \leq \inf \left\{\left\lceil P_{\lambda_{r}, j_{\infty}}\right\rceil(\mathbf{u}) \mid \mathbf{u} \in L D(\Omega)\right\}$, since $\lambda_{r} \leq \inf \left(P_{0, j}\right)_{A L}$ (see (2.9), (2.10) and (3.1)). In view of Lemma 5, there exists $\widehat{\boldsymbol{\sigma}} \in W^{n}(\Omega, \operatorname{div})$ such that $\operatorname{div} \widehat{\boldsymbol{\sigma}}=-\mathbf{f}$ in $\Omega$ and $\boldsymbol{\beta}_{B}(\widehat{\boldsymbol{\sigma}})=\mathbf{g}$ on $\Gamma_{1}$. Then, by the Green formula [4, Theorem 2] and Proposition 12, there exists $0<k_{\widehat{\sigma}}<+\infty$ such that

$$
\begin{aligned}
0 \leq \inf _{\mathbf{u} \in L D}\left\{\left\lceil P_{\lambda_{r}, j_{\infty}}\right\rceil(\mathbf{u})\right\} \\
=-\sup \left\{\int_{\Omega} \lambda_{r} \widehat{\boldsymbol{\sigma}}: \boldsymbol{\varepsilon}(\mathbf{u}) d x-\int_{\operatorname{Fr} \Omega} \boldsymbol{\beta}_{B}\left(\lambda_{r} \widehat{\boldsymbol{\sigma}}\right) \cdot \gamma_{B}(\mathbf{u}) d s\right. \\
\left.\quad-\Theta_{\lambda_{r}}\left(\boldsymbol{\varepsilon}(\mathbf{u}), \gamma_{B}(\mathbf{u})\right) \mid \mathbf{u} \in L D(\Omega)\right\} \\
=-\Theta_{\lambda_{r}}^{\#}\left(\lambda_{r} \widehat{\boldsymbol{\sigma}}\right)=-\operatorname{cl}_{A_{k_{\widehat{\sigma}}}} Q\left(\lambda_{r} \widehat{\boldsymbol{\sigma}}\right),
\end{aligned}
$$

where $\mathbf{u}^{0}=\mathbf{0}$ on $\Gamma_{0}$. Therefore, by (4.18), there exists a net $\left\{\boldsymbol{\sigma}_{t}\right\}_{t \in T} \subset$ $W^{n}(\Omega, \operatorname{div})$ such that $\boldsymbol{\beta}_{B}\left(\boldsymbol{\sigma}_{t}\right)=\lambda_{r} \mathbf{g}$ on $\Gamma_{1}, \boldsymbol{\sigma}_{t}(x) \in \mathcal{K}(x)$ for $d x$-a.e. $x \in$ $\Omega$ and every $t \in T$, and $\left\langle\left(\boldsymbol{\sigma}_{t}-\lambda_{r} \widehat{\boldsymbol{\sigma}}\right),(\mathbf{w}, \mathbf{p})\right\rangle_{2} \rightarrow 0$ for every $(\mathbf{w}, \mathbf{p}) \in$ $L^{1}\left(\Omega, \mathbb{E}_{s}^{n}\right) \times L^{1}(\operatorname{Fr} \Omega)^{n}$ with $\mathbf{p}_{\mid \Gamma_{0}}=\mathbf{0}$. Then, by the Green formula [4, (3.9)], $\int_{\Omega}\left(\lambda_{r} \mathbf{f}+\operatorname{div} \boldsymbol{\sigma}_{t}\right) \cdot \mathbf{u} d x \rightarrow 0$ for every $\mathbf{u} \in L D(\Omega)$ with $\boldsymbol{\gamma}_{B}(\mathbf{u})=\mathbf{0}$ on $\Gamma_{0}$.

Due to (4.25) and Proposition 12 the net $\left\{\left\|\operatorname{div} \boldsymbol{\sigma}_{t}\right\|_{L^{n}}\right\}_{t \in T}$ is bounded by $k_{\widehat{\boldsymbol{\sigma}}}<+\infty$. The set $\left\{\mathbf{u} \in L D(\Omega) \mid \boldsymbol{\gamma}_{B}(\mathbf{u})_{\mid \Gamma_{0}}=\mathbf{0}\right\}$ is dense in $\left[L^{n /(n-1)}(\Omega)^{n}\right.$, $\left.\|\cdot\|_{L^{n /(n-1)}}\right]$, because $C_{0}^{1}(\Omega)^{n}$ is dense in $L^{n /(n-1)}(\Omega)^{n}$. The space $\left[\mathrm{cl}_{\|\cdot\|_{L^{n}}}\right.$ $\left.\left(B_{L^{n}}\left(0,\left\|\lambda_{r} \mathbf{f}\right\|_{L^{n}}+k_{\widehat{\boldsymbol{\sigma}}}\right)\right), \sigma\left(L^{n}, L^{n /(n-1)}\right)\right]$ is compact and $L^{n}(\Omega)^{n}$ endowed with the topology $\sigma\left(L^{n}(\Omega)^{n},\left\{\mathbf{u} \in L D(\Omega) \mid \boldsymbol{\gamma}_{B}(\mathbf{u})_{\mid \Gamma_{0}}=\mathbf{0}\right\}\right)$ is a Hausdorff space. Then

$$
\int_{\Omega}\left(\lambda_{r} \mathbf{f}+\operatorname{div} \boldsymbol{\sigma}_{t}\right) \cdot \mathbf{w} d x \rightarrow 0 \quad \forall \mathbf{w} \in L^{n /(n-1)}(\Omega)^{n},
$$

since among all Hausdorff topologies compact topologies are minimal (see [9, Corollary 3.1.14]). Therefore, by the Mazur lemma, there exists a sequence $\left\{\boldsymbol{\sigma}_{m}\right\}_{m \in \mathbb{N}} \subset W^{n}(\Omega, \operatorname{div})$ such that $\boldsymbol{\beta}_{B}\left(\boldsymbol{\sigma}_{m}\right)=\lambda_{r} \mathbf{g}$ on $\Gamma_{1}, \boldsymbol{\sigma}_{m}(x) \in \mathcal{K}(x)$ for $d x$-a.e. $x \in \Omega$ and every $m \in \mathbb{N}$. Moreover, $\left\|\lambda_{r} \mathbf{f}+\operatorname{div} \boldsymbol{\sigma}_{m}\right\|_{L^{n}(\Omega)^{n}} \rightarrow 0$.

The following criterion of coercivity of $\left\lceil P_{\lambda, j}\right\rceil$ is formulated for any elasticplastic potential $j$ (which satisfies (2.5), (2.7) and Assumptions 1-2).

Proposition 14 (cf. [2] and [16]). Assume that $\bar{\lambda}_{r}$ satisfies Assumption 4, where $\lambda_{r}$ is replaced by $\bar{\lambda}_{r}$. If $\inf \left(P_{0, j}\right)_{A L}>\bar{\lambda}_{r}>\lambda_{r} \geq 0$ then $\inf \left\{\left\lceil P_{\lambda_{r}, j}\right\rceil(\mathbf{u}) \mid \mathbf{u} \in L D(\Omega)\right\}>-\infty$. The converse holds in the following form: if $\inf \left\{\left\lceil P_{\lambda_{r}, j}\right\rceil(\mathbf{u}) \mid \mathbf{u} \in L D(\Omega)\right\}>-\infty$, then $\inf \left(P_{0, j}\right)_{A L} \geq \lambda_{r}$. More- 
over, if $\inf \left(P_{0, j}\right)_{A L}>\bar{\lambda}_{r}>\lambda_{r} \geq 0$ then any sequence $\left\{\mathbf{u}_{m}\right\}_{m \in \mathbb{N}} \subset B D(\Omega)$ such that

$$
\inf _{\mathbf{z}}\left\{\left\|\mathbf{u}_{m}+\mathbf{z}\right\|_{B D} \mid \mathbf{z} \in B D(\Omega) \text { and } \boldsymbol{\varepsilon}(\mathbf{z})=\mathbf{0}\right\} \rightarrow+\infty
$$

satisfies $\lim _{m \rightarrow \infty}\left(F_{\lambda_{r}}\left(\mathbf{u}_{m}\right)+G_{j}\left(\varepsilon\left(\mathbf{u}_{m}\right)\right)\right)=+\infty$ (cf. (2.10)).

Proof. Step 1. Let $\inf \left(P_{0, j}\right)_{A L}>\bar{\lambda}_{r}>\lambda_{r} \geq 0$. By Proposition 13 there exist sequences $\left\{\boldsymbol{\sigma}_{m}\right\}_{m \in \mathbb{N}} \subset W^{n}(\Omega$, div $)$ and $\left\{r_{m}\right\}_{m \in \mathbb{N}} \subset \mathbb{R}$ such that $\left\|\boldsymbol{\sigma}_{m}\right\|_{L^{\infty}}<r_{m}, \beta_{B}\left(\boldsymbol{\sigma}_{m}\right)=\bar{\lambda}_{r} \mathbf{g}$ on $\Gamma_{1}$ and $\boldsymbol{\sigma}_{m}(x) \in \mathcal{K}(x)$ for $d x$-a.e. $x \in \Omega$. Moreover, $\left\|\bar{\lambda}_{r} \mathbf{f}+\operatorname{div} \boldsymbol{\sigma}_{m}\right\|_{L^{n}(\Omega)^{n}} \rightarrow 0$. Then, by (2.6), we obtain

$$
\begin{aligned}
& \inf \left\{-\int_{\Omega}\left(-\operatorname{div} \boldsymbol{\sigma}_{m}\right) \cdot \mathbf{u} d x-\bar{\lambda}_{r} \int_{\Gamma_{1}} \mathbf{g} \cdot \boldsymbol{\gamma}_{B}(\mathbf{u}) d s\right. \\
& \left.\quad+\int_{\Omega} j(x, \boldsymbol{\varepsilon}(\mathbf{u})) d x \mid \mathbf{u} \in L D(\Omega), \gamma_{B}(\mathbf{u})=\mathbf{0} \text { on } \Gamma_{0}\right\} \\
& =\inf \left\{-\int_{\Omega} \boldsymbol{\sigma}_{m}: \boldsymbol{\varepsilon}(\mathbf{u}) d x+\int_{\Omega} j(x, \boldsymbol{\varepsilon}(\mathbf{u})) d x \mid\right. \\
& \geq-\sup \left\{\int_{\Omega} \boldsymbol{\sigma}_{m}: \mathbf{w} d x-\int_{\Omega} j(x, \mathbf{w}) d x \mid \mathbf{w} \in L^{1}\left(\Omega, \mathbb{E}_{s}^{n}\right)\right\} \\
& =-\int_{\Omega} j^{*}\left(x, \boldsymbol{\sigma}_{m}\right) d x>-c_{r_{m}}>-\infty \quad \forall m \in \mathbb{N} .
\end{aligned}
$$

Step 2. If $d s\left(\Gamma_{0}\right)=0$, then for every $m \in \mathbb{N}$ and every $\widetilde{\mathbf{u}} \in L D(\Omega)$ such that $\varepsilon(\widetilde{\mathbf{u}})=\mathbf{0}$ in $\Omega$, we have $L_{-\operatorname{div}} \boldsymbol{\sigma}_{m}, \bar{\lambda}_{r} \mathbf{g}(\widetilde{\mathbf{u}})=0$ where

$$
L_{-\operatorname{div} \boldsymbol{\sigma}_{m}, \bar{\lambda}_{r} \mathbf{g}}(\mathbf{u}) \equiv \int_{\Omega}\left(-\operatorname{div} \boldsymbol{\sigma}_{m}\right) \cdot \mathbf{u} d x+\bar{\lambda}_{r} \int_{\Gamma_{1}} \mathbf{g} \cdot \boldsymbol{\gamma}_{B}(\mathbf{u}) d s
$$

for every $\mathbf{u} \in L D(\Omega)$. Indeed, if $L_{-\operatorname{div}} \boldsymbol{\sigma}_{m}, \bar{\lambda}_{r} \mathbf{g}(\widetilde{\mathbf{u}}) \neq 0$ and $\varepsilon(\widetilde{\mathbf{u}})=\mathbf{0}$ in $\Omega$, then (4.28) is not bounded from below, where $j$ is replaced by $j_{\infty}$.

Step 3. Let $\left\{\left(\widetilde{\mathbf{f}}_{m}, \widetilde{\mathbf{g}}_{m}\right)\right\}_{m \in \mathbb{N}} \subset L^{n}(\Omega)^{n} \times L^{\infty}\left(\Gamma_{1}\right)^{n}$ and $L_{\widetilde{\mathbf{f}}_{m}, \widetilde{\mathbf{g}}_{m}}(\widetilde{\mathbf{u}})=0$ for every $(m, \widetilde{\mathbf{u}}) \in \mathbb{N} \times L D(\Omega)$ with $\varepsilon(\widetilde{\mathbf{u}})=\mathbf{0}$ in $\Omega$, where $L_{\widetilde{\mathbf{f}}_{m}, \widetilde{\mathbf{g}}_{m}}$ is defined by (4.29). Moreover, suppose $\left\|\widetilde{\mathbf{f}}_{m}-\widetilde{\mathbf{f}}_{0}\right\|_{L^{n}(\Omega)^{n}} \rightarrow 0$ and $\left\|\widetilde{\mathbf{g}}_{m}-\widetilde{\mathbf{g}}_{0}\right\|_{L^{\infty}\left(\Gamma_{1}\right)^{n}} \rightarrow 0$. Then $L_{\widetilde{\mathbf{f}}_{0}, \widetilde{\mathbf{g}}_{0}}(\widetilde{\mathbf{u}})=0$ for every $\widetilde{\mathbf{u}} \in L D(\Omega)$ such that $\boldsymbol{\varepsilon}(\widetilde{\mathbf{u}})=\mathbf{0}$ in $\Omega$.

Step 4. We define a continuous linear function

$$
\begin{aligned}
& W^{n}(\Omega, \operatorname{div}) \ni \boldsymbol{\sigma} \mapsto \Phi_{a}(\boldsymbol{\sigma}) \\
& \quad=\left(-\operatorname{div} \boldsymbol{\sigma}, \boldsymbol{\beta}_{B}(\boldsymbol{\sigma})_{\mid \Gamma_{1}}\right) \in L^{n}(\Omega)^{n} \times L^{\infty}\left(\Gamma_{1}\right)^{n},
\end{aligned}
$$

where the spaces $W^{n}(\Omega, \operatorname{div}), L^{n}(\Omega)^{n}$ and $L^{\infty}\left(\Gamma_{1}\right)^{n}$ are endowed with the norms $\|\cdot\|_{W^{n}(\Omega, \text { div })},\|\cdot\|_{L^{n}}$ and $\|\cdot\|_{L^{\infty}}$. Let $\boldsymbol{\sigma}_{1}, \boldsymbol{\sigma}_{2} \in W^{n}(\Omega, \operatorname{div})$ and

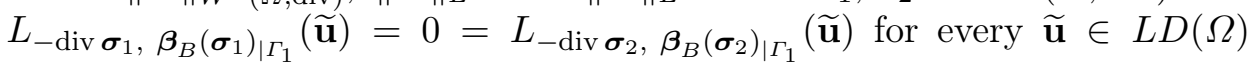


with $\varepsilon(\widetilde{\mathbf{u}})=\mathbf{0}$ in $\Omega$. Then, for all $a, b \in \mathbb{R}, \boldsymbol{\sigma}_{3}=a \boldsymbol{\sigma}_{1}+b \boldsymbol{\sigma}_{2}$ satisfies the

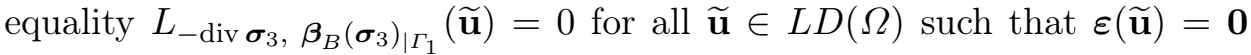
in $\Omega$.

Let $\left\{\tilde{\boldsymbol{\sigma}}_{m}\right\} \subset W^{n}(\Omega, \operatorname{div})$ with $\left\|\widetilde{\boldsymbol{\sigma}}_{m}-\widetilde{\boldsymbol{\sigma}}_{0}\right\|_{W^{n}(\Omega, \text { div })} \rightarrow 0$. Moreover, suppose $L_{-\operatorname{div}} \boldsymbol{\sigma}_{m}, \boldsymbol{\beta}_{B}\left(\boldsymbol{\sigma}_{m}\right)_{\mid \Gamma_{1}}(\widetilde{\mathbf{u}})=0$ for every $m \in \mathbb{N}$ and every $\widetilde{\mathbf{u}} \in L D(\Omega)$ such that $\varepsilon(\widetilde{\mathbf{u}})=\mathbf{0}$ in $\Omega$. Then, by the continuity of $\Phi_{a}$ we obtain

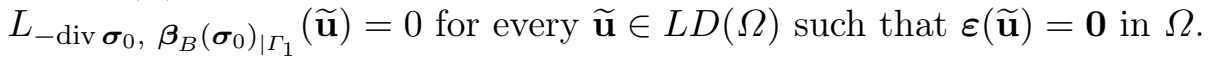

Step 5. There exists a closed (in $\left.\|\cdot\|_{W^{n}(\Omega, \text { div })}\right)$ subspace $W_{L}^{n}(\Omega, \operatorname{div})$ of $W^{n}(\Omega$, div $)$ such that for every $\widehat{\boldsymbol{\sigma}} \in W_{L}^{n}(\Omega, \operatorname{div})$ we have $L_{-\operatorname{div}} \widehat{\boldsymbol{\sigma}}, \boldsymbol{\beta}_{B}(\widehat{\boldsymbol{\sigma}})_{\mid \Gamma_{1}}(\widetilde{\mathbf{u}})$ $=0$ for every $\widetilde{\mathbf{u}} \in L D(\Omega)$ with $\varepsilon(\widetilde{\mathbf{u}})=\mathbf{0}$ in $\Omega$. Moreover, by Lemma 5 , for every $(\widetilde{\mathbf{f}}, \widetilde{\mathbf{g}}) \subset L^{n}(\Omega)^{n} \times L^{\infty}\left(\Gamma_{1}\right)^{n}$ such that $L_{\widetilde{\mathbf{f}}, \widetilde{\mathbf{g}}}(\widetilde{\mathbf{u}})=0$ (if $\boldsymbol{\varepsilon}(\widetilde{\mathbf{u}})=\mathbf{0}$, for $\widetilde{\mathbf{u}} \in L D(\Omega))$, there exists $\widetilde{\boldsymbol{\sigma}} \in W_{L}^{n}(\Omega, \operatorname{div})$ and $\Phi_{a}(\widetilde{\boldsymbol{\sigma}})=(\widetilde{\mathbf{f}}, \widetilde{\mathbf{g}})$.

In view of Steps 3 and $4, \Phi_{a \mid W_{L}^{n}(\Omega, \text { div })}$ is a continuous linear functional defined on the Banach space $\left[W_{L}^{n}(\Omega, \operatorname{div}),\|\cdot\|_{W^{n}(\Omega, \text { div })}\right]$. Moreover, $\Phi_{a \mid W_{L}^{n}(\Omega, \operatorname{div})}$ is a surjection on the Banach space

$$
\left\{(\mathbf{f}, \mathbf{g}) \in L^{n}(\Omega)^{n} \times L^{\infty}\left(\Gamma_{1}\right)^{n} \mid L_{\mathbf{f}, \mathbf{g}}(\widetilde{\mathbf{u}})=0 \forall \widetilde{\mathbf{u}} \in L D(\Omega), \boldsymbol{\varepsilon}(\widetilde{\mathbf{u}})=\mathbf{0}\right\}
$$

endowed with the norm $\|\cdot\|_{L^{n}} \times\|\cdot\|_{L^{\infty}}$.

Step 6. Let $d s\left(\Gamma_{0}\right)>0$. By the first part of Lemma $5, \Phi_{a}$ is a surjection on the Banach space $L^{n}(\Omega)^{n} \times L^{\infty}\left(\Gamma_{1}\right)^{n}$. Then, in view of the interior mapping principle [7, Theorem II.2.1], there exist open balls int $B_{L^{n}(\Omega) \times L^{\infty}\left(\Gamma_{1}\right)}\left(\mathbf{z}, r_{a}\right)$ $\subset L^{n}(\Omega)^{n} \times L^{\infty}\left(\Gamma_{1}\right)^{n}$ and int $B_{W^{n}(\Omega, \text { div })}\left(\mathbf{0}, r_{b}\right) \subset W^{n}(\Omega$, div $)$ such that

$$
\operatorname{int} B_{L^{n}(\Omega) \times L^{\infty}\left(\Gamma_{1}\right)}\left(\mathbf{z}, r_{a}\right) \subset \Phi_{a}\left(\operatorname{int} B_{W^{n}(\Omega, \operatorname{div})}\left(\mathbf{0}, r_{b}\right)\right) \text {. }
$$

There exists $r_{c}>0\left(r_{b}<r_{c}<\infty\right)$ such that $-\mathbf{z} \in \Phi_{a}\left(\operatorname{int} B_{W^{n}(\Omega, \operatorname{div})}\left(\mathbf{0}, r_{c}\right)\right)$. Then int $B_{L^{n}(\Omega) \times L^{\infty}\left(\Gamma_{1}\right)}\left(\mathbf{0},\left(r_{a} / 2\right)\right) \subset \Phi_{a}\left(\operatorname{int} B_{W^{n}(\Omega, \operatorname{div})}\left(\mathbf{0}, r_{c}\right)\right)$, since $\Phi_{a}$ is linear and $r_{c}>r_{b}$.

Step 7. By Step 5 and the interior mapping principle [7], there exist open sets int $B_{(4.31)}\left(\mathbf{0}, \widehat{r}_{a}\right)($ a ball in the space $(4.31))$ and int $B_{W_{L}^{n}(\Omega, \operatorname{div})}\left(\mathbf{0}, \widehat{r}_{b}\right) \subset$ $W_{L}^{n}(\Omega, \operatorname{div})$ with

$$
\operatorname{int} B_{(4.31)}\left(\mathbf{0}, \widehat{r}_{a}\right) \subset \Phi_{a}\left(\operatorname{int} B_{W_{L}^{n}(\Omega, \operatorname{div})}\left(\mathbf{0}, \widehat{r}_{b}\right)\right) .
$$

Step 8. Let $\left\{\boldsymbol{\sigma}_{m}\right\}_{m \in \mathbb{N}}$ be as in Step 1. Then $\lambda_{r} \mathbf{g}=\boldsymbol{\beta}_{B}\left(\boldsymbol{\sigma}_{m} \lambda_{r} / \bar{\lambda}_{r}\right)$ on $\Gamma_{1}$, $\left\|\lambda_{r} \mathbf{f}+\operatorname{div}\left(\boldsymbol{\sigma}_{m} \lambda_{r} / \bar{\lambda}_{r}\right)\right\|_{L^{n}(\Omega)^{n}} \rightarrow 0$ and $\boldsymbol{\sigma}_{m} \in \mathcal{K}(x)$ for $d x$-a.e. $x \in \Omega$.

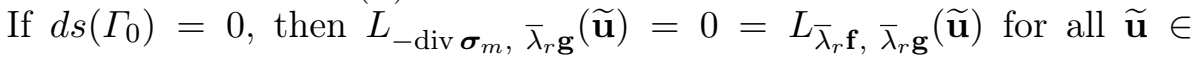
$L D(\Omega)$ with $\varepsilon(\widetilde{\mathbf{u}})=\mathbf{0}$ in $\Omega$ (see Steps 2 and 3). Therefore, there exists a sequence $\left\{\widetilde{\boldsymbol{\sigma}}_{m}\right\}_{m \in \mathbb{N}} \subset W^{n}(\Omega$, div $)$ such that $\boldsymbol{\beta}_{B}\left(\widetilde{\boldsymbol{\sigma}}_{m}\right)=\mathbf{0}$ on $\Gamma_{1}$ and $\operatorname{div}\left(\widetilde{\boldsymbol{\sigma}}_{m}\left(\bar{\lambda}_{r}-\lambda_{r}\right) / \bar{\lambda}_{r}\right)=-\lambda_{r} \mathbf{f}-\operatorname{div}\left(\boldsymbol{\sigma}_{m} \lambda_{r} / \bar{\lambda}_{r}\right)$ in $\Omega$ for every $m \in \mathbb{N}$. In view of (4.32) we can assume that $\left\|\widetilde{\boldsymbol{\sigma}}_{m}\right\|_{W^{n}(\Omega \text {,div })} \rightarrow 0$ as $m \rightarrow \infty$, because $\left\|\operatorname{div} \widetilde{\boldsymbol{\sigma}}_{m}\right\|_{L^{n}(\Omega)^{n}} \rightarrow 0$ and $\Phi_{a}$ is a linear function. 
Similarly, if $d s\left(\Gamma_{0}\right)>0$, then there exists a sequence $\left\{\widetilde{\boldsymbol{\sigma}}_{m}\right\}_{m \in \mathbb{N}}$ such that $\boldsymbol{\beta}_{B}\left(\widetilde{\boldsymbol{\sigma}}_{m}\right)=\mathbf{0}$ on $\Gamma_{1}, \operatorname{div}\left(\widetilde{\boldsymbol{\sigma}}_{m}\left(\bar{\lambda}_{r}-\lambda_{r}\right) / \bar{\lambda}_{r}\right)=-\lambda_{r} \mathbf{f}-\operatorname{div}\left(\boldsymbol{\sigma}_{m} \lambda_{r} / \bar{\lambda}_{r}\right)$ in $\Omega$ for every $m \in \mathbb{N}$ and $\left\|\widetilde{\boldsymbol{\sigma}}_{m}\right\|_{W^{n}(\Omega, \text { div })} \rightarrow 0$ as $m \rightarrow \infty$. Therefore, by (4.28),

$$
\begin{array}{r}
\inf \left\{-L_{\lambda_{r} \mathbf{f}, \lambda_{r} \mathbf{g}(\mathbf{u})+\int_{\Omega} j(x, \boldsymbol{\varepsilon}(\mathbf{u})) d x \mid} \mathbf{u} \in L D(\Omega), \boldsymbol{\gamma}_{B}(\mathbf{u})=\mathbf{0} \text { on } \Gamma_{0}\right\} \\
\geq \inf \left\{-L_{-\operatorname{div}} \widetilde{\boldsymbol{\sigma}}_{m}\left(\bar{\lambda}_{r}-\lambda_{r}\right) / \bar{\lambda}_{r}, \mathbf{0}(\mathbf{u})+\left(\bar{\lambda}_{r}-\lambda_{r}\right)\left(\bar{\lambda}_{r}\right)^{-1} \int_{\Omega} j(x, \boldsymbol{\varepsilon}(\mathbf{u})) d x \mid\right. \\
\left.\mathbf{u} \in L D(\Omega), \gamma_{B}(\mathbf{u})=\mathbf{0} \text { on } \Gamma\right\}+\inf \left\{-L_{-\operatorname{div}} \boldsymbol{\sigma}_{m} \lambda_{r} / \bar{\lambda}_{r}, \lambda_{r} \mathbf{g}(\mathbf{u})\right. \\
\left.+\left(\bar{\lambda}_{r}\right)^{-1} \lambda_{r} \int_{\Omega} j(x, \boldsymbol{\varepsilon}(\mathbf{u})) d x \mid \mathbf{u} \in L D(\Omega), \gamma_{B}(\mathbf{u})=\mathbf{0} \text { on } \Gamma_{0}\right\} \\
\geq-\left(\bar{\lambda}_{r}-\lambda_{r}\right)\left(\bar{\lambda}_{r}\right)^{-1} \int_{\Omega} j^{*}\left(x, \widetilde{\boldsymbol{\sigma}}_{m}\right) d x-\left(\bar{\lambda}_{r}\right)^{-1} \lambda_{r} \int_{\Omega} j^{*}\left(x, \boldsymbol{\sigma}_{m}\right) d x
\end{array}
$$

for all $m \in \mathbb{N}$ (in both cases). There exists $m_{0} \in \mathbb{N}$ with $\left\|\widetilde{\boldsymbol{\sigma}}_{m_{0}}\right\|_{L^{\infty}(\Omega)^{n}}<r_{1}$ (cf. Assumption 2). Then $-\int_{\Omega} j^{*}\left(x, \widetilde{\boldsymbol{\sigma}}_{m_{0}}\right) d x \geq-k d x(\Omega)$ and $-\int_{\Omega} j^{*}\left(x, \boldsymbol{\sigma}_{m_{0}}\right) d x$ $>-c_{r_{m_{0}}}>-\infty$, where $d x(\Omega)$ is the Lebesgue measure of $\Omega$.

Step 9. In view of Definition $1, j_{\infty} \geq j$. Since $\inf \left\{\left\lceil P_{\lambda_{r}, j}\right\rceil(\mathbf{u}) \mid \mathbf{u} \in\right.$ $L D(\Omega)\}>-\infty$, it follows that $\inf \left\{\left\lceil P_{\lambda_{r}, j_{\infty}}\right\rceil(\mathbf{u}) \mid \mathbf{u} \in L D(\Omega)\right\}>-\infty$ and $\inf \left(P_{0, j}\right)_{A L} \geq \lambda_{r}$. In the case when $\lambda_{r}>0$, the last part of the proposition follows from the first part with $\lambda_{r}$ replaced by $\left(\bar{\lambda}_{r}+\lambda_{r}\right) / 2$. If $\lambda_{r}=0$, the last part follows directly from Definition 1 and Assumption 2.

In view of Definition 1, Assumption 2 and the fact that $\Omega$ is bounded, the following result holds.

Theorem 15 (see [3, Theorem 18]). Assume that $\mathbf{u}^{0}=\mathbf{0}$ and $\bar{\lambda}_{r}$ satisfies Assumption 4. If $0 \leq \lambda_{r}<\bar{\lambda}_{r}<\inf \left(P_{0, j}\right)_{A L}$, then the l.s.c. regularization of

$$
B D(\Omega) \ni \mathbf{u} \mapsto\left\lceil R P_{\lambda_{r}, j}\right\rceil(\mathbf{u})=F_{\lambda_{r}}(\mathbf{u})+G_{j}(\varepsilon(\mathbf{u})) \in \mathbb{R} \cup\{\infty\}
$$

in the weak $*^{*} B D(\Omega)$ topology is $B D(\Omega) \ni \mathbf{u} \mapsto\left\lceil R P_{\lambda_{r}, j}^{* *}\right\rceil(\mathbf{u}) \in \mathbb{R} \cup\{+\infty\}$, i.e., $\left\lceil R P_{\lambda_{r}, j}^{* *}\right\rceil$ is the largest l.s.c. minorant less than (4.34).

Corollary 16. Let $\mathbf{u}^{0}=\mathbf{0}$ on $\Gamma_{0}$ and let $\lambda_{s}$ be the supremum of all $\lambda_{r}$ satisfying Assumption 4. Then $\min \left(\lambda_{s}, \inf \left(P_{0, j}\right)_{A L}\right)=\min \left(\lambda_{s}, \inf \left(R P_{0, j}^{* *}\right)_{A L}\right)$ (cf. (3.1) and (3.2)).

Proof. Suppose $\min \left(\lambda_{s}, \inf \left(P_{0, j}\right)_{A L}\right)>\hat{\lambda}$. We have $\inf \left(P_{0, j}\right)_{A L} \geq \hat{\lambda}$ if and only if $\inf \left\{\left\lceil P_{\widehat{\lambda}, j_{\infty}}\right\rceil(\mathbf{u}) \mid \mathbf{u} \in B D(\Omega)\right\} \geq 0$. By Theorem 15, the 1.s.c. regularization of $\mathbf{u} \mapsto\left\lceil P_{\widehat{\lambda}, j_{\infty}}\right\rceil(\mathbf{u}) \in \mathbb{R} \cup\{\infty\}$ in the weak ${ }^{*} B D(\Omega)$ topology equals $\left\lceil R P_{\widehat{\lambda}, j_{\infty}}^{* *}\right\rceil$. Then $\inf \left\{\left\lceil R P_{\widehat{\lambda}, j_{\infty}}^{* *}\right\rceil(\mathbf{u}) \mid \mathbf{u} \in B D(\Omega)\right\} \geq 0$. Therefore, $\inf \left(R P_{0, j}^{* *}\right)_{A L} \geq \hat{\lambda}$. 
5. The proof of regularity of displacement solutions. In this section it is proved that every minimum of $\left\lceil R P_{\lambda, j}^{* *}\right\rceil$ belongs to the space $L D(\Omega)$ (if the criterion of regularity of displacements is satisfied, cf. Theorem 1 ). Below $\mathbf{u}^{0}=\mathbf{0}$ on $\Gamma_{0}$. Moreover, it is not assumed that the set $\mathcal{K}(x)$ is bounded for each $x \in \bar{\Omega}$.

The functional $\mathbb{B}_{\lambda}^{j, \mathbf{f}}: \mathbf{Y}^{1}(\bar{\Omega}) \rightarrow \mathbb{R} \cup\{\infty\}$ is defined by

$$
\begin{aligned}
\mathbb{B}_{\lambda}^{j, \mathbf{f}}\left(\varepsilon(\mathbf{u})_{\mid \bar{\Omega}}\right) & \equiv-\lambda\left\langle\boldsymbol{\sigma}_{L},\left(\varepsilon(\mathbf{u})_{\mid \Omega}, \boldsymbol{\gamma}_{B}^{I}(\mathbf{u})\right)\right\rangle_{2}-\lambda \int_{\Gamma_{1}} \boldsymbol{\beta}_{B}\left(\boldsymbol{\sigma}_{L}\right) \boldsymbol{\gamma}_{B}^{I}(\mathbf{u}) d s \\
& +\int_{\Gamma_{0}} I_{\left\{\boldsymbol{\gamma}_{B}^{I}(\mathbf{u}) \otimes_{s} \boldsymbol{\nu}=0\right\}}\left(-\boldsymbol{\gamma}_{B}^{I}(\mathbf{u}) \otimes_{s} \boldsymbol{\nu}\right) d s+\int_{\Omega} j(x, \boldsymbol{\varepsilon}(\mathbf{u})) d x
\end{aligned}
$$

if $\mathbf{u}_{\mid \Omega} \in L D(\Omega)$ and $\mathbf{u}_{\mid \Omega_{1}-\bar{\Omega}}=\mathbf{0}$, and $\mathbb{B}_{\lambda}^{j, \mathbf{f}}\left(\varepsilon(\mathbf{u})_{\mid \bar{\Omega}}\right) \equiv+\infty$ otherwise. By (2.9) we have $\mathbb{B}_{\lambda}^{j, \mathbf{f}}\left(\varepsilon(\mathbf{u})_{\mid \bar{\Omega}}\right)=F_{\lambda}\left(\mathbf{u}_{\mid \Omega}\right)+G_{j}\left(\varepsilon\left(\mathbf{u}_{\mid \Omega}\right)\right)$ if $\mathbf{u}_{\mid \Omega} \in L D(\Omega)$ and $\mathbf{u}_{\mid \Omega_{1}-\bar{\Omega}}=\mathbf{0}$. The extension $\tilde{\mathbf{Y}}^{1}(\bar{\Omega})$ of $\mathbf{Y}^{1}(\bar{\Omega})$ is given by

$$
\begin{array}{r}
\widetilde{\mathbf{Y}}^{1}(\bar{\Omega}) \equiv\left\{\left(\mathbf{z},-\boldsymbol{\gamma}_{B}^{I}(\mathbf{u}) \otimes_{s} \boldsymbol{\nu}\right) \in \operatorname{span}\left(\varepsilon(B D(\Omega)), L^{1}\left(\Omega, \mathbb{E}_{s}^{n}\right)\right) \times \mathbf{Y}^{1}(\bar{\Omega})_{\mid \operatorname{Fr} \Omega} \mid\right. \\
\exists \mathbf{w} \in L^{1}\left(\Omega, \mathbb{E}_{s}^{n}\right), \quad \exists \widetilde{\mathbf{u}} \in B D(\Omega) \text { such that } \mathbf{z}=\mathbf{w} d x+\boldsymbol{\varepsilon}(\widetilde{\mathbf{u}}) \\
\text { and } \left.\boldsymbol{\gamma}_{B}^{I}(\mathbf{u}) \otimes_{s} \boldsymbol{\nu}=\boldsymbol{\gamma}_{B}(\widetilde{\mathbf{u}}) \otimes_{s} \boldsymbol{\nu}\right\}
\end{array}
$$

(cf. [4]). The bilinear form between $\widetilde{\mathbf{Y}}^{1}(\bar{\Omega})$ and $W^{n}(\Omega$, div) is given by

$$
\left\langle\left(\mathbf{z},-\boldsymbol{\gamma}_{B}^{I}(\mathbf{u}) \otimes_{s} \boldsymbol{\nu}\right), \boldsymbol{\sigma}\right\rangle_{1} \equiv \int_{\Omega} \boldsymbol{\sigma}: \mathbf{z}-\int_{\operatorname{Fr} \Omega} \boldsymbol{\beta}_{B}(\boldsymbol{\sigma}) \cdot \gamma_{B}^{I}(\mathbf{u}) d s
$$

for $\boldsymbol{\sigma} \in W^{n}(\Omega, \operatorname{div})$ and $\left(\mathbf{z},-\gamma_{B}^{I}(\mathbf{u}) \otimes_{s} \boldsymbol{\nu}\right) \in \widetilde{\mathbf{Y}}^{1}(\bar{\Omega})$. A net $\left\{\mathbf{M}_{t}\right\}_{t \in T} \subset \widetilde{\mathbf{Y}}^{1}(\bar{\Omega})$ is convergent to $\mathbf{M}_{0}$ in $\sigma\left(\tilde{\mathbf{Y}}^{1}(\bar{\Omega}), W^{n}(\Omega, \operatorname{div})\right)$ if $\left\langle\mathbf{M}_{t}, \boldsymbol{\sigma}\right\rangle_{1} \rightarrow\left\langle\mathbf{M}_{0}, \boldsymbol{\sigma}\right\rangle_{1}$ for all $\boldsymbol{\sigma} \in W^{n}\left(\Omega_{1}\right.$, div). The extension of $\mathbb{B}_{\lambda}^{j, \mathbf{f}}$ on the space $\widetilde{\mathbf{Y}}^{1}(\bar{\Omega})$ is

$$
\begin{aligned}
\widetilde{\mathbb{B}}_{\lambda}^{j, \mathbf{f}}\left(\mathbf{z},-\boldsymbol{\gamma}_{B}(\mathbf{u}) \otimes_{s} \boldsymbol{\nu}\right) \\
\equiv-\lambda\left\langle\left(\mathbf{z},-\boldsymbol{\gamma}_{B}(\mathbf{u}) \otimes_{s} \boldsymbol{\nu}\right), \boldsymbol{\sigma}_{L}\right\rangle_{1}-\lambda \int_{\Gamma_{1}} \boldsymbol{\beta}_{B}\left(\boldsymbol{\sigma}_{L}\right) \boldsymbol{\gamma}_{B}(\mathbf{u}) d s \\
\quad+\int_{\Gamma_{0}} I_{\left\{\boldsymbol{\gamma}_{B}(\mathbf{u}) \otimes_{s} \boldsymbol{\nu}=\mathbf{0}\right\}}\left(-\boldsymbol{\gamma}_{B}(\mathbf{u}) \otimes_{s} \boldsymbol{\nu}\right) d s+\int_{\Omega} j(x, \mathbf{z}) d x
\end{aligned}
$$

if $\mathbf{z}=\mathbf{w} d x+\varepsilon(\mathbf{u})$ with $(\mathbf{w}, \mathbf{u}) \in L^{1}\left(\Omega, \mathbb{E}_{s}^{n}\right) \times L D(\Omega)$, and $\widetilde{\mathbb{B}}_{\lambda}^{j, \mathbf{f}}\left(\mathbf{z},-\boldsymbol{\gamma}_{B}(\mathbf{u}) \otimes_{s} \boldsymbol{\nu}\right)$ $\equiv+\infty$ otherwise.

Because of the duality between $\mathbf{Y}^{1}(\bar{\Omega})$ and $W^{n}(\Omega$, div), we obtain

$$
\begin{aligned}
\left(\mathbb{B}_{\lambda}^{j, \mathbf{f}}\right)^{\#}(\boldsymbol{\sigma}) \equiv \sup \left\{\left\langle\boldsymbol{\sigma},\left(\boldsymbol{\varepsilon}(\mathbf{u})_{\mid \Omega}, \boldsymbol{\gamma}_{B}^{I}(\mathbf{u})\right)\right\rangle_{2}-\mathbb{B}_{\lambda}^{j, \mathbf{f}}\left(\boldsymbol{\varepsilon}(\mathbf{u})_{\mid \bar{\Omega}}\right) \mid\right. & \\
\mathbf{u} & \left.\in B D\left(\Omega_{1}\right), \mathbf{u}_{\mid \Omega} \in L D(\Omega), \mathbf{u}_{\mid \Omega_{1}-\bar{\Omega}}=\mathbf{0}\right\}
\end{aligned}
$$


for every $\boldsymbol{\sigma} \in W^{n}(\Omega$, div $)$, and

$$
\begin{aligned}
& (5.6) \quad\left(\mathbb{B}_{\lambda}^{j, \mathbf{f}}\right)^{\# \#}\left(\boldsymbol{\varepsilon}(\mathbf{u})_{\mid \bar{\Omega}}\right) \\
& \equiv \sup _{\boldsymbol{\sigma}}\left\{\int_{\Omega} \boldsymbol{\sigma}: \boldsymbol{\varepsilon}(\mathbf{u})-\int_{\operatorname{Fr} \Omega} \boldsymbol{\beta}_{B}(\boldsymbol{\sigma}) \cdot \boldsymbol{\gamma}_{B}^{I}(\mathbf{u}) d s-\left(\mathbb{B}_{\lambda}^{j, \mathbf{f}}\right)^{\#}(\boldsymbol{\sigma}) \mid \boldsymbol{\sigma} \in W^{n}(\Omega, \operatorname{div})\right\}
\end{aligned}
$$

for every $\mathbf{u} \in B D\left(\Omega_{1}\right)$ such that $\mathbf{u}_{\mid \Omega_{1}-\bar{\Omega}}=\mathbf{0}$.

Similarly, by the duality between $\widetilde{\mathbf{Y}}^{1}(\bar{\Omega})$ and $W^{n}(\Omega$, div) we define functionals $\left(\widetilde{\mathbb{B}}_{\lambda}^{j, \mathbf{f}}\right) \#$ and $\left(\widetilde{\mathbb{B}}_{\lambda}^{j, \mathbf{f}}\right) \# \#: \widetilde{\mathbf{Y}}^{1}(\bar{\Omega}) \rightarrow \mathbb{R} \cup\{+\infty\}$ by

$$
\begin{aligned}
\left(\widetilde{\mathbb{B}}_{\lambda}^{j, \mathbf{f}}\right)^{\#}(\boldsymbol{\sigma}) \equiv & \sup \left\{\int_{\Omega} \boldsymbol{\sigma}: \mathbf{z} d x-\int_{\operatorname{Fr} \Omega} \boldsymbol{\beta}_{B}(\boldsymbol{\sigma}) \cdot \boldsymbol{\gamma}_{B}(\mathbf{u}) d s\right. \\
& \left.-\widetilde{\mathbb{B}}_{\lambda}^{j, \mathbf{f}}\left(\mathbf{z},-\boldsymbol{\gamma}_{B}(\mathbf{u}) \otimes_{s} \boldsymbol{\nu}\right) \mid \mathbf{z} \in L^{1}\left(\Omega, \mathbb{E}_{s}^{n}\right), \mathbf{u} \in L D(\Omega)\right\}
\end{aligned}
$$

for $\boldsymbol{\sigma} \in W^{n}(\Omega, \operatorname{div})$, and

$$
\equiv \sup \left\{\int_{\Omega} \boldsymbol{\sigma}: \mathbf{z}-\int_{\operatorname{Fr} \Omega} \boldsymbol{\beta}_{B}(\boldsymbol{\sigma}) \cdot \gamma_{B}^{I}(\mathbf{u}) d s-\left(\widetilde{\mathbb{B}}_{\lambda}^{j, \mathbf{f}}\right)^{\#}(\boldsymbol{\sigma}) \mid \boldsymbol{\sigma} \in W^{n}(\Omega, \operatorname{div})\right\} .
$$

Proposition 17. The explicit form of $\left(\widetilde{\mathbb{B}}_{\lambda}^{j, \mathbf{f}}\right)^{\#}$ is

$$
\begin{aligned}
\left(\widetilde{\mathbb{B}}_{\lambda}^{j, \mathbf{f}}\right)^{\#}(\boldsymbol{\sigma})= & \int_{\Omega} j^{*}\left(x, \boldsymbol{\sigma}+\lambda \boldsymbol{\sigma}_{L}\right) d x \\
& +\int_{\Gamma_{1}} I_{\left\{\boldsymbol{\sigma}+\lambda \boldsymbol{\sigma}_{L} \mid \boldsymbol{\beta}_{B}\left(\boldsymbol{\sigma}+\lambda \boldsymbol{\sigma}_{L}\right)=\lambda \mathbf{g}\right\}}\left(\boldsymbol{\sigma}+\lambda \boldsymbol{\sigma}_{L}\right) d s
\end{aligned}
$$

for every $\boldsymbol{\sigma} \in W^{n}(\Omega, \operatorname{div})$. If $\lambda_{L}$ satisfies Assumption 5 , then $\left(\widetilde{\mathbb{B}}_{\lambda_{L}}^{j, \mathbf{f}}\right)^{\# \#}(\varepsilon(\mathbf{u})$, $\left.-\gamma_{B}^{I}(\mathbf{u}) \otimes_{s} \boldsymbol{\nu}\right)=\widetilde{\mathbb{B}}_{\lambda_{L}}^{j, \mathbf{f}}\left(\varepsilon(\mathbf{u}),-\gamma_{B}^{I}(\mathbf{u}) \otimes_{s} \boldsymbol{\nu}\right) \quad$ for every $\mathbf{u} \in L D(\Omega)$ such that $\gamma_{B}^{I}(\mathbf{u})=\mathbf{0}$ on $\Gamma_{0}$.

Proof. By [15, Theorem 3A] and formulae (5.4), (5.7), we have

$$
\begin{aligned}
\left(\widetilde{\mathbb{B}}_{\lambda}^{j, \mathbf{f}}\right)^{\#}(\boldsymbol{\sigma})= & \sup \left\{\int_{\Omega}\left(\boldsymbol{\sigma}+\lambda \boldsymbol{\sigma}_{L}\right): \mathbf{z} d x\right. \\
& -\int_{\operatorname{Fr} \Omega} \boldsymbol{\beta}_{B}\left(\boldsymbol{\sigma}+\lambda \boldsymbol{\sigma}_{L}\right) \cdot \boldsymbol{\gamma}_{B}(\mathbf{u}) d s+\int_{\Gamma_{1}} \boldsymbol{\beta}_{B}\left(\lambda \boldsymbol{\sigma}_{L}\right) \cdot \boldsymbol{\gamma}_{B}(\mathbf{u}) d s \\
& -\int_{\Gamma_{0}} I_{\left\{\boldsymbol{\gamma}_{B}(\mathbf{u}) \otimes_{s} \boldsymbol{\nu}=\mathbf{0}\right\}}\left(-\boldsymbol{\gamma}_{B}(\mathbf{u}) \otimes_{s} \boldsymbol{\nu}\right) d s-\int_{\Omega} j(x, \mathbf{z}) d x \\
\mathbf{z} & \left.=\mathbf{w}+\boldsymbol{\varepsilon}(\mathbf{u}), \text { where } \mathbf{w} \in L^{1}\left(\Omega, \mathbb{E}_{s}^{n}\right) \text { and } \mathbf{u} \in L D(\Omega)\right\}
\end{aligned}
$$




$$
\begin{array}{r}
=\sup \left\{\int_{\Omega}\left(\boldsymbol{\sigma}+\lambda \boldsymbol{\sigma}_{L}\right): \mathbf{w} d x-\int_{\Omega} j(x, \mathbf{w}) d x \mid \mathbf{w} \in L^{1}\left(\Omega, \mathbb{E}_{s}^{n}\right)\right\} \\
+\sup \left\{-\int_{\operatorname{Fr} \Omega} \boldsymbol{\beta}_{B}\left(\boldsymbol{\sigma}+\lambda \boldsymbol{\sigma}_{L}\right) \cdot \boldsymbol{\gamma}_{B}(\mathbf{u}) d s+\int_{\Gamma_{1}} \lambda \mathbf{g} \cdot \boldsymbol{\gamma}_{B}(\mathbf{u}) d s \mid\right. \\
\left.\gamma_{B}(\mathbf{u}) \in L^{1}(\operatorname{Fr} \Omega)^{n} \text { and } \boldsymbol{\gamma}_{B}(\mathbf{u})=\mathbf{0} \text { on } \Gamma_{0}\right\},
\end{array}
$$

which yields (5.9) for every $\boldsymbol{\sigma} \in W^{n}\left(\Omega\right.$, div), because $\gamma_{B}$ is a surjection on $L^{1}(\operatorname{Fr} \Omega)^{n}$.

The space $W^{n}(\Omega$, div $)$ is PCU-stable, so by [5, Theorem 1] we get, for every $\mathbf{u} \in L D(\Omega)$,

$$
\begin{aligned}
& \left(\widetilde{\mathbb{B}}_{\lambda_{L}}^{j, \mathbf{f}}\right) \# \#\left(\varepsilon(\mathbf{u}),-\gamma_{B}(\mathbf{u}) \otimes_{s} \boldsymbol{\nu}\right)=\sup \left\{\int_{\Omega}\left(\boldsymbol{\sigma}+\lambda_{L} \boldsymbol{\sigma}_{L}\right): \varepsilon(\mathbf{u}) d x\right. \\
& -\int_{\Omega} j^{*}\left(x, \boldsymbol{\sigma}+\lambda_{L} \boldsymbol{\sigma}_{L}\right) d x-\int_{\Gamma_{0}}^{\Omega} \boldsymbol{\beta}_{B}\left(\boldsymbol{\sigma}+\lambda_{L} \boldsymbol{\sigma}_{L}\right) \cdot \gamma_{B}(\mathbf{u}) d s \\
& -\int_{\Gamma_{1}} \boldsymbol{\beta}_{B}\left(\boldsymbol{\sigma}+\lambda_{L} \boldsymbol{\sigma}_{L}\right) \cdot \gamma_{B}(\mathbf{u}) d s-\int_{\Gamma_{1}} I_{\left\{\boldsymbol{\beta}_{B}\left(\boldsymbol{\sigma}+\lambda_{L} \boldsymbol{\sigma}_{L}\right)=\lambda_{L} \mathbf{g}\right\}}\left(\boldsymbol{\sigma}+\lambda_{L} \boldsymbol{\sigma}_{L}\right) d s \\
& \left.\boldsymbol{\sigma} \in W^{n}(\Omega, \operatorname{div}), \boldsymbol{\beta}_{B}\left(\boldsymbol{\sigma}+\lambda_{L} \boldsymbol{\sigma}_{L}\right)(x) \in \mathcal{K}(x) \cdot \boldsymbol{\nu}(x) \text { for } d s \text {-a.e. } x \in \operatorname{Fr} \Omega\right\} \\
& -\lambda_{L}\left(\int_{\Omega} \boldsymbol{\sigma}_{L}: \boldsymbol{\varepsilon}(\mathbf{u}) d x-\int_{\operatorname{Fr} \Omega} \boldsymbol{\beta}_{B}\left(\boldsymbol{\sigma}_{L}\right) \cdot \gamma_{B}(\mathbf{u}) d s\right) \\
& =-\lambda_{L}\left(\int_{\Omega} \boldsymbol{\sigma}_{L}: \boldsymbol{\varepsilon}(\mathbf{u}) d x-\int_{\operatorname{Fr} \Omega} \boldsymbol{\beta}_{B}\left(\boldsymbol{\sigma}_{L}\right) \cdot \gamma_{B}^{I}(\mathbf{u}) d s+\int_{\Gamma_{1}} \mathbf{g} \cdot \boldsymbol{\gamma}_{B}^{I}(\mathbf{u}) d s\right) \\
& +\int_{\Gamma_{0}} j_{\infty}\left(x,-\gamma_{B}^{I}(\mathbf{u}) \otimes_{s} \boldsymbol{\nu}\right) d s+\int_{\Omega} j(x, \boldsymbol{\varepsilon}(\mathbf{u})) d x
\end{aligned}
$$

(see [4, Proposition 25], 4, (7.60)] and formulae (2.5), (2.6)).

LEMma 18 (see [3, Lemma 6]). For every $\boldsymbol{\sigma} \in W^{n}(\Omega$, div) we have $\left(\widetilde{\mathbb{B}}_{\lambda}^{j, \mathbf{f}}\right) \#(\boldsymbol{\sigma}) \geq\left(\mathbb{B}_{\lambda}^{j, \mathbf{f}}\right) \#(\boldsymbol{\sigma})$ and for every $\mathbf{M} \in \mathbf{Y}^{1}(\bar{\Omega})$ we have $\left(\widetilde{\mathbb{B}}_{\lambda}^{j, \mathbf{f}}\right)^{\# \#}(\mathbf{M}) \leq$ $\left(\mathbb{B}_{\lambda}^{j, \mathbf{f}}\right) \# \#(\mathbf{M})$, since $\mathbf{Y}^{1}(\bar{\Omega}) \subset \tilde{\mathbf{Y}}^{1}(\bar{\Omega})$.

LEMMA 19 (cf. [3, Lemma 8]). If $\lambda_{L}$ satisfies Assumption 5 then, for every $\mathbf{u} \in L D(\Omega)$ such that $\gamma_{B}^{I}(\mathbf{u})_{\mid \Gamma_{0}}=\mathbf{0}$, we have

$$
\begin{aligned}
\left(\widetilde{\mathbb{B}}_{\lambda_{L}}^{j, \mathbf{f}}\right) \# \#\left(\varepsilon(\mathbf{u}),-\gamma_{B}(\mathbf{u}) \otimes_{s} \boldsymbol{\nu}\right) & =\left(\mathbb{B}_{\lambda_{L}}^{j, \mathbf{f}}\right) \# \#\left(\varepsilon(\mathbf{u}),-\gamma_{B}(\mathbf{u}) \otimes_{s} \boldsymbol{\nu}\right) \\
& =\left(\mathbb{B}_{\lambda_{L}}^{j, \mathbf{f}}\right)\left(\varepsilon(\mathbf{u}),-\gamma_{B}(\mathbf{u}) \otimes_{s} \boldsymbol{\nu}\right)
\end{aligned}
$$

LEMMA 20 (see [3, Lemma 9]). For every $\boldsymbol{\sigma} \in W^{n}(\Omega$, div) and every $\boldsymbol{\sigma}_{s} \in W^{n}(\Omega, \operatorname{div})$ such that $\operatorname{div} \boldsymbol{\sigma}_{s}=\mathbf{0}$, we have $\left(\mathbb{B}_{\lambda}^{j, \mathbf{f}}\right)^{\#}(\boldsymbol{\sigma})=\left(\mathbb{B}_{\lambda}^{j, \mathbf{f}}\right)^{\#}\left(\boldsymbol{\sigma}+\boldsymbol{\sigma}_{s}\right)$.

If there exists $r_{2}>0\left(r_{2}<+\infty\right)$ such that for every $x \in \bar{\Omega}, \mathcal{K}(x) \subset$ $B_{\mathbb{E}_{s}^{n}}\left(0, r_{2}\right)$, and if $0 \leq \lambda_{L}<\lambda_{r}<\inf \left(P_{0, j}\right)_{A L}$ and Assumption 4 holds, then, by Proposition 14 and [4, Theorem 14], Assumptions 5 and 6 hold. 
Proposition 21. If Assumptions 5 and 6 hold, then $\inf \left\{\mathbb{B}_{\lambda_{L}}^{j, \mathbf{f}}(\mathbf{M}) \mid \mathbf{M} \in\right.$ $\left.\mathbf{Y}^{1}(\bar{\Omega})\right\}=\inf \left\{\widetilde{\mathbb{B}}_{\lambda_{L}}^{j, \mathbf{f}}(\mathbf{M}) \mid \mathbf{M} \in \widetilde{\mathbf{Y}}^{1}(\bar{\Omega})\right\}$

Proof. By formulae (5.7), (5.9), (3.3), 4, (6.7) and (6.8)] and Assumption 5 , we get

$$
\begin{aligned}
& \sup \left\{-\widetilde{\mathbb{B}}_{\lambda_{L}}^{j, \mathbf{f}}(\mathbf{M}) \mid \mathbf{M} \in \widetilde{\mathbf{Y}}^{1}(\bar{\Omega})\right\}=\left(\widetilde{\mathbb{B}}_{\lambda_{L}}^{j, \mathbf{f}}\right) \#(\mathbf{0}) \\
& =-\left\lceil P_{\lambda_{L}, j}^{*}\right\rceil\left(\lambda_{L} \boldsymbol{\sigma}_{L}\right)=\inf \left\{-\left\lceil P_{\lambda_{L}, j}^{*}\right\rceil(\boldsymbol{\sigma}) \mid \boldsymbol{\sigma} \in W^{n}(\Omega, \operatorname{div})\right\} .
\end{aligned}
$$

Moreover, by Assumption 6 and (5.1), we obtain

$$
\begin{aligned}
& \inf \left\{-\left\lceil P_{\lambda_{L}, j}^{*}\right\rceil(\boldsymbol{\sigma}) \mid \boldsymbol{\sigma} \in W^{n}(\Omega, \operatorname{div})\right\} \\
= & \sup \left\{-\left\lceil P_{\lambda_{L}, j}\right\rceil(\mathbf{u}) \mid \mathbf{u} \in B D(\Omega)\right\}=\sup \left\{-\mathbb{B}_{\lambda_{L}}^{j, \mathbf{f}}(\mathbf{M}) \mid \mathbf{M} \in \mathbf{Y}^{1}(\bar{\Omega})\right\} .
\end{aligned}
$$

Let us recall that the l.s.c. regularization of the functional $\mathbb{B}$ in the topology $\tau$, denoted by $\operatorname{cl}_{\tau} \mathbb{B}$, is the largest $\tau$-l.s.c. minorant less than $\mathbb{B}$.

Corollary 22. If Assumptions 5 and 6 hold, then, by Proposition 21,

$$
\begin{aligned}
\inf \left\{\operatorname{cl}_{\sigma\left(\mathbf{Y}^{1}(\bar{\Omega}), W^{n}(\Omega, \operatorname{div})\right)} \mathbb{B}_{\lambda_{L}}^{j, \mathbf{f}}(\mathbf{M}) \mid \mathbf{M} \in \mathbf{Y}^{1}(\bar{\Omega})\right\} \\
\quad=\inf \left\{\operatorname{cl}_{\sigma\left(\widetilde{\mathbf{Y}}^{1}(\bar{\Omega}), W^{n}(\Omega, \operatorname{div})\right)} \widetilde{\mathbb{B}}_{\lambda_{L}}^{j, \mathbf{f}}(\mathbf{M}) \mid \mathbf{M} \in \widetilde{\mathbf{Y}}^{1}(\bar{\Omega})\right\},
\end{aligned}
$$

because $\mathbf{0} \in W^{n}(\Omega$, div $)$.

Consider the following problem:

$$
\left(\widetilde{P}_{\lambda, j}\right) \quad \text { find } \inf \left\{\widetilde{\mathbb{B}}_{\lambda}^{j, \mathbf{f}}(\mathbf{M}) \mid \mathbf{M} \in \widetilde{\mathbf{Y}}^{1}(\bar{\Omega})\right\} .
$$

The limit analysis problem $\left(\widetilde{P}_{0, j}\right)_{A L}$, defined in $(3.7)$, is connected with $\left(\widetilde{P}_{\lambda, j}\right)$.

Definition 2. Suppose that $U$ is a locally convex space, $U^{*}$ its topological dual, $\langle\cdot, \cdot\rangle_{U}$ the bilinear pairing over $U \times U^{*}$ and $\Phi$ a mapping of $U$ into $\mathbb{R} \cup\{\infty\}$. If $\Phi(\psi)<\infty$ then we denote by $\partial \Phi(\psi)$ (where $\psi \in U$ ) the set

$$
\left\{\psi^{*} \in U^{*} \mid \forall \varrho \in U,\left\langle\varrho-\psi, \psi^{*}\right\rangle_{U}+\Phi(\psi) \leq \Phi(\varrho)\right\} .
$$

In the proof below, Assumptions 5, 6 and 7 hold.

Proof of Theorem 1. Step 1. Let $\widetilde{\mathbf{u}}$ be a minimum of $\left\lceil R P_{\lambda_{L}, j}^{* *}\right\rceil$. By Theorem 15 and formulae (2.9), (2.10), (3.4), (3.5), (3.6), (5.1), the functional $\left\lceil R P_{\lambda_{L}, j}^{* *}\right\rceil$ is the 1.s.c. regularization of $\mathbb{B}_{\lambda_{L}}^{j, \mathbf{f}}$ in the weak* $B D(\Omega)$ topology. Let $\widetilde{\mathbf{u}}_{1} \in B D\left(\Omega_{1}\right)$ with $\widetilde{\mathbf{u}}_{1 \mid \Omega}=\widetilde{\mathbf{u}}$ and $\widetilde{\mathbf{u}}_{1 \mid \Omega_{1}-\bar{\Omega}}=\mathbf{0}$. Then

$$
\begin{aligned}
& \operatorname{cl}_{\sigma\left(\mathbf{Y}^{1}(\bar{\Omega}), W^{n}(\Omega, \operatorname{div})\right)} \mathbb{B}_{\lambda_{L}}^{j, \mathbf{f}}\left(\varepsilon\left(\widetilde{\mathbf{u}}_{1}\right)_{\mid \bar{\Omega}}\right) \\
& =\inf \left\{\operatorname{cl}_{\sigma\left(\widetilde{\mathbf{Y}}^{1}(\bar{\Omega}), W^{n}(\Omega, \operatorname{div})\right)} \widetilde{\mathbb{B}}_{\lambda_{L}}^{j, \mathbf{f}}(\mathbf{M}) \mid \mathbf{M} \in \widetilde{\mathbf{Y}}^{1}(\bar{\Omega})\right\}
\end{aligned}
$$


(cf. Corollary 22). For every $\mathbf{M} \in \mathbf{Y}^{1}(\bar{\Omega})$ we have $\mathbb{B}_{\lambda_{L}}^{j, \mathbf{f}}(\mathbf{M})=\widetilde{\mathbb{B}}_{\lambda_{L}}^{j, \mathbf{f}}(\mathbf{M})$, so for every $\mathbf{M} \in \mathbf{Y}^{1}(\bar{\Omega})$,

$$
\operatorname{cl}_{\sigma\left(\mathbf{Y}^{1}(\bar{\Omega}), W^{n}(\Omega, \operatorname{div})\right)} \mathbb{B}_{\lambda_{L}}^{j, \mathbf{f}}(\mathbf{M}) \geq \operatorname{cl}_{\sigma\left(\widetilde{\mathbf{Y}}^{1}(\bar{\Omega}), W^{n}(\Omega, \operatorname{div})\right)} \widetilde{\mathbb{B}}_{\lambda_{L}}^{j, \mathbf{f}}(\mathbf{M}) .
$$

The restriction of the measure $\varepsilon\left(\widetilde{\mathbf{u}}_{1}\right)_{\mid \bar{\Omega}}$ to the open set $\Omega$ is denoted by $\varepsilon\left(\widetilde{\mathbf{u}}_{1}\right)_{\mid \Omega}$. Because of (5.17) and Corollary 22, the point $\varepsilon\left(\widetilde{\mathbf{u}}_{1}\right)_{\mid \bar{\Omega}}=\left(\varepsilon\left(\widetilde{\mathbf{u}}_{1}\right)_{\mid \Omega}\right.$, $\left.-\gamma_{B}^{I}\left(\widetilde{\mathbf{u}}_{1}\right) \otimes_{s} \boldsymbol{\nu}\right) \in \widetilde{\mathbf{Y}}^{1}(\bar{\Omega})$ is a minimum of the function $\operatorname{cl}_{\sigma\left(\widetilde{\mathbf{Y}}^{1}(\bar{\Omega}), W^{n}(\Omega, \operatorname{div})\right)} \widetilde{\mathbb{B}}_{\lambda_{L}}^{j, \mathbf{f}}$ on $\widetilde{\mathbf{Y}}^{1}(\bar{\Omega})$. By Definition 2 we get $\mathbf{0} \in \partial\left(\operatorname{cl}_{\sigma\left(\widetilde{\mathbf{Y}}^{1}(\bar{\Omega}), W^{n}(\Omega, \operatorname{div})\right)} \widetilde{\mathbb{B}}_{\lambda_{L}}^{j, \mathbf{f}}\right)\left(\varepsilon\left(\widetilde{\mathbf{u}}_{1}\right)_{\mid \bar{\Omega}}\right)$, where $\mathbf{0} \in W^{n}(\Omega$, div $)$. Then $\left(\varepsilon\left(\widetilde{\mathbf{u}}_{1}\right)_{\mid \bar{\Omega}}\right) \in \partial\left(\mathrm{cl}_{\sigma\left(\widetilde{\mathbf{Y}}^{1}(\bar{\Omega}), W^{n}(\Omega, \operatorname{div})\right)} \widetilde{\mathbb{B}}_{\lambda_{L}}^{j, \mathbf{f}}\right) \#(\mathbf{0})$ (see [8, Chapter 1, Corollary 5.2]). By (5.7) we have $\varepsilon\left(\widetilde{\mathbf{u}}_{1}\right)_{\mid \bar{\Omega}}=\left(\varepsilon\left(\widetilde{\mathbf{u}}_{1}\right)_{\mid \Omega}\right.$, $\left.-\gamma_{B}^{I}\left(\widetilde{\mathbf{u}}_{1}\right) \otimes_{s} \boldsymbol{\nu}\right) \in \partial\left(\widetilde{\mathbb{B}}_{\lambda_{L}}^{j, \mathbf{f}}\right) \#(\mathbf{0})$. Then by Definition 2 we get

$$
\left\langle\left(\varepsilon\left(\widetilde{\mathbf{u}}_{1}\right)_{\mid \Omega},-\gamma_{B}^{I}\left(\widetilde{\mathbf{u}}_{1}\right) \otimes_{s} \nu\right), \boldsymbol{\sigma}-\mathbf{0}\right\rangle_{1}+\left(\widetilde{\mathbb{B}}_{\lambda_{L}}^{j, \mathbf{f}}\right) \#(\mathbf{0}) \leq\left(\widetilde{\mathbb{B}}_{\lambda_{L}}^{j, \mathbf{f}}\right) \#(\boldsymbol{\sigma})
$$

for every $\boldsymbol{\sigma} \in W^{n}(\Omega$, div) (cf. (5.3) and (4.3)).

Step 2. If $0<\lambda_{2}<\inf \left(\widetilde{P}_{0, j}\right)_{A L}$ then, by (3.7), (5.4) and Assumption 2, we have $\inf \left\{\widetilde{\mathbb{B}}_{\lambda_{2}}^{j_{\infty}, \mathbf{f}}(\mathbf{M}) \mid \mathbf{M} \in \widetilde{\mathbf{Y}}^{1}(\bar{\Omega})\right\}=0$. Moreover, $\sup \left\{-\widetilde{\mathbb{B}}_{\lambda_{2}}^{j_{\infty}, \mathbf{f}}(\mathbf{M}) \mid \mathbf{M} \in\right.$ $\left.\widetilde{\mathbf{Y}}^{1}(\bar{\Omega})\right\}=\left(\widetilde{\mathbb{B}}_{\lambda_{2}}^{j_{\infty}, \mathbf{f}}\right) \#(\mathbf{0})$. Therefore, $\left(\widetilde{\mathbb{B}}_{\lambda_{2}}^{j_{\infty}, \mathbf{f}}\right) \#(\mathbf{0})=0$.

Step 3. There exists $\lambda_{1}$ such that $\lambda_{L}<\lambda_{1}<\inf \left(\widetilde{P}_{0, j}\right)_{A L}$. By Step 2 we have $\left(\widetilde{\mathbb{B}}_{\lambda_{1}}^{j_{\infty}, \mathbf{f}}\right) \#(\mathbf{0})=0$. Then by $(5.9), \lambda_{1} \boldsymbol{\sigma}_{L}(x) \in \mathcal{K}(x)$ for $d x$-a.e. $x \in \Omega$. By (2.5) and Assumption 2 we get

$$
B_{\mathbb{E}_{s}^{n}}\left(\lambda_{L} \boldsymbol{\sigma}_{L}(x), \frac{\lambda_{1}-\lambda_{L}}{\lambda_{1}} r_{1}\right) \subset \mathcal{K}(x)
$$

for $d x$-a.e. $x \in \Omega$.

Step 4. Due to Assumption 3, $\Gamma_{1}=\operatorname{Fr} \Omega \cap \mathcal{C}$, where $\mathcal{C}=\operatorname{clint} \mathcal{C} \subset \Omega_{1}$ is a closed Caccioppoli set and $d s(\operatorname{Fr} \Omega \cap \operatorname{Fr} \mathcal{C})=0$. Let $\mathcal{O}_{\Gamma_{0}}=\Omega_{1}-\mathcal{C}$. Then $d s\left(\Gamma_{0}-\left(\operatorname{Fr} \Omega \cap \mathcal{O}_{\Gamma_{0}}\right)\right)=0$ and $d s\left(\left(\operatorname{Fr} \Omega \cap \mathcal{O}_{\Gamma_{0}}\right)-\Gamma_{0}\right)=0$. We define $\Gamma_{0}^{\prime}=\operatorname{Fr} \Omega \cap \mathcal{O}_{\Gamma_{0}}$. Then for every $t \in \mathbb{N}$ there exists an open set $\Omega_{t}^{\prime}$ such that $\Omega_{t}^{\prime} \subset \mathcal{O}_{\Gamma_{0}}, \Omega_{t}^{\prime} \subset \subset \Omega_{1}, d x\left(\Omega_{t}^{\prime}\right)<1 / 2 t$ and $\left\{x \in \Gamma_{0}^{\prime} \mid \boldsymbol{\gamma}_{B}^{I}\left(\widetilde{\mathbf{u}}_{1}\right)(x) \neq \mathbf{0}\right\} \subset \Omega_{t}^{\prime}$ for $d s$-a.e. $x \in \operatorname{Fr} \Omega$.

Step 5. Suppose the singular part $\left(\varepsilon\left(\widetilde{\mathbf{u}}_{1}\right)_{\mid \Omega}\right)_{s}$ of the measure $\varepsilon\left(\widetilde{\mathbf{u}}_{1}\right)_{\mid \Omega}$ is not 0 or $d s\left(\left\{x \in \Gamma_{0}^{\prime} \mid \boldsymbol{\gamma}_{B}^{I}\left(\widetilde{\mathbf{u}}_{1}\right)(x) \neq \mathbf{0}\right\}\right)>0$. Then there exists $\zeta>0$ such that $\left\|\left(\varepsilon\left(\widetilde{\mathbf{u}}_{1}\right)_{\mid \Omega}\right)_{s}\right\|_{\mathbb{M}_{b}}+\int_{\Gamma_{0}^{\prime}}\left\|\left(\gamma_{B}^{I}\left(\widetilde{\mathbf{u}}_{1}\right) \otimes_{s} \boldsymbol{\nu}\right)(x)\right\|_{\mathbb{E}_{s}^{n}} d s>\zeta$. Therefore, for every $t \in \mathbb{N}$ there exist open sets $\Omega_{t}^{\prime \prime} \subset \subset \Omega$ and $\Omega_{t}^{0} \equiv \Omega_{t}^{\prime \prime} \cup \Omega_{t}^{\prime} \subset \subset \Omega_{1}$ such that the Lebesgue measure of $\Omega_{t}^{0}$ (equal to $d x\left(\Omega_{t}^{0}\right)$ ) is less than $1 / t$ and $\left\|\left(\varepsilon\left(\widetilde{\mathbf{u}}_{1}\right)_{\mid \Omega_{t}^{\prime \prime}}\right)_{s}\right\|_{\mathbb{M}_{b}}+\int_{\Gamma_{0}^{\prime}}\left\|\left(\gamma_{B}^{I}\left(\widetilde{\mathbf{u}}_{1}\right) \otimes_{s} \boldsymbol{\nu}\right)(x)\right\|_{\mathbb{E}_{s}^{n}} d s>\frac{1}{2} \zeta$. The existence of the sequence $\left\{\Omega_{t}^{\prime \prime}\right\}_{t \in \mathbb{N}}$ satisfying the above conditions follows from the regularity of the measure $\varepsilon\left(\widetilde{\mathbf{u}}_{1}\right)_{\mid \Omega}$. 
Then for every $t \in \mathbb{N}$ there exists $\varphi_{t} \in C_{0}^{1}\left(\Omega_{1}, \mathbb{E}_{s}^{n}\right)$ such that $\varphi_{t \mid \Omega_{1}-\Omega_{t}^{0}}$ $=0$,

$$
\left\|\boldsymbol{\varphi}_{t}(x)\right\|_{\mathbb{E}_{s}^{n}}<\frac{\lambda_{1}-\lambda_{L}}{2 \lambda_{1}} r_{1} \quad \forall x \in \Omega_{t}^{0}
$$

and

$$
\left\langle\left(\varepsilon\left(\widetilde{\mathbf{u}}_{1}\right)_{\mid \Omega},-\gamma_{B}^{I}\left(\widetilde{\mathbf{u}}_{1}\right) \otimes_{s} \boldsymbol{\nu}\right), \boldsymbol{\varphi}_{t \mid \bar{\Omega}}\right\rangle_{1}>\frac{1}{4} \zeta \frac{\lambda_{1}-\lambda_{L}}{2 \lambda_{1} n^{2}} r_{1},
$$

since $\left\|\left(\varepsilon\left(\widetilde{\mathbf{u}}_{1}\right)_{\mid \Omega_{t}^{\prime \prime}}\right)_{s}\right\|_{\mathbb{M}_{b}}+\int_{\Gamma_{0}^{\prime}}\left\|\left(\gamma_{B}^{I}\left(\widetilde{\mathbf{u}}_{1}\right) \otimes_{s} \boldsymbol{\nu}\right)(x)\right\|_{\mathbb{E}_{s}^{n}} d s>\frac{1}{2} \zeta$, and

$$
\begin{aligned}
& \left\|\varepsilon\left(\widetilde{\mathbf{u}}_{1}\right)_{\mid \Omega_{t}^{0}}\right\|_{\mathbb{M}_{b}}=\sup \left\{\left\langle\varepsilon\left(\widetilde{\mathbf{u}}_{1}\right)_{\mid \Omega_{t}^{0}}, \widetilde{\varphi}\right\rangle_{\mathbb{M}_{b} \times C\left(\Omega_{t}^{0}, \mathbb{E}_{s}^{n}\right)} \mid\right. \\
& \left.\quad \widetilde{\varphi} \in C_{0}^{1}\left(\Omega_{t}^{0}, \mathbb{E}_{s}^{n}\right) \text { and } \forall x \in \Omega_{t}^{0}, \forall i, j \in\{1, \ldots, n\},\left|\widetilde{\varphi}_{i j}(x)\right| \leq 1\right\}
\end{aligned}
$$

(cf. definition of $\|\cdot\|_{\mathbb{E}_{s}^{n}}$ in Section 2 and [4, (3.18)]).

Step 6. By Assumption 7 there exists $\delta>0$ such that

$$
\begin{aligned}
\left|\left(\widetilde{\mathbb{B}}_{\lambda_{L}}^{j, \mathbf{f}}\right) \#\left(\boldsymbol{\varphi}_{t \mid \Omega}\right)-\left(\widetilde{\mathbb{B}}_{\lambda_{L}}^{j, \mathbf{f}}\right) \#(\mathbf{0})\right| & <\delta \frac{\lambda_{1}-\lambda_{L}}{2 \lambda_{1}} r_{1} d x\left(\Omega_{t}^{0} \cap \Omega\right) \\
& <\delta \frac{\lambda_{1}-\lambda_{L}}{2 \lambda_{1}} r_{1} \frac{1}{t}
\end{aligned}
$$

for every $t \in \mathbb{N}$, since $\boldsymbol{\varphi}_{t}(x)+\lambda_{L} \boldsymbol{\sigma}_{L}(x) \in \mathcal{K}(x)$ for $d x$-a.e. $x \in \Omega$ and $\boldsymbol{\varphi}_{t \mid \Omega}+\lambda_{L} \boldsymbol{\sigma}_{L} \in L^{\infty}\left(\Omega, \mathbb{E}_{s}^{n}\right)$ (cf. (5.20), (5.19)). By (5.18) we get

$$
\left\langle\left(\varepsilon\left(\widetilde{\mathbf{u}}_{1}\right)_{\mid \Omega},-\boldsymbol{\gamma}_{B}^{I}\left(\widetilde{\mathbf{u}}_{1}\right) \otimes_{s} \boldsymbol{\nu}\right), \boldsymbol{\varphi}_{t \mid \bar{\Omega}}\right\rangle_{1} \leq\left|\left(\widetilde{\mathbb{B}}_{\lambda_{L}}^{j, \mathbf{f}}\right) \#\left(\boldsymbol{\varphi}_{t \mid \Omega}\right)-\left(\widetilde{\mathbb{B}}_{\lambda_{L}}^{j, \mathbf{f}}\right)^{\#}(\mathbf{0})\right|
$$

for every $t \in \mathbb{N}$. Then, due to (5.21) and (5.23), we have a contradiction, because $\delta \frac{\lambda_{1}-\lambda_{L}}{2 \lambda_{1}} r_{1} \frac{1}{t} \rightarrow 0$ as $t \rightarrow \infty$.

Due to (3.7), we have proved the regularity result if the stress solution belongs to the interior of the set of admissible stresses, $d x$-almost everywhere on $\Omega$.

\section{References}

[1] G. Anzellotti and M. Giaquinta, Convex functionals and partial regularity, Arch. Ration. Mech. Anal. 102 (1988), 243-272.

[2] J. L. Bojarski, The relaxation of Signorini problems in Hencky plasticity, I: Threedimensional solid, Nonlinear Anal. 29 (1997), 1091-1116.

[3] -, General method of regularization. I: Functionals defined on BD space, Appl. Math. (Warsaw) 31 (2004), 175-199.

[4] -, Regularity of displacement solutions in Hencky plasticity. I: The extremal relation, ibid. 38 (2011), 259-293.

[5] G. Bouchitté and M. Valadier, Integral representation of convex functionals on a space of measures, J. Funct. Anal. 80 (1988), 398-420.

[6] M. Costabel, M. Dauge and S. Nicaise, Singularities of Maxwell interface problems, Math. Model. Numer. Anal. 33 (1999), 627-649. 
[7] N. Dunford and J. T. Schwartz, Linear Operators, Part I, Interscience, New York, 1958.

[8] I. Ekeland and R. Temam, Convex Analysis and Variational Problems, NorthHolland, Amsterdam, 1976.

[9] R. Engelking, General Topology, PWN-Polish Sci. Publ., Warszawa, 1977.

[10] R. Hardt and D. Kinderlehrer, Elastic plastic deformation, Appl. Math. Optim. (3) 10 (1983), 203-246.

[11] R. Kohn and R. Temam, Dual spaces of stresses and strains with applications to Hencky plasticity, Appl. Math. Optim. 10 (1983), 1-35.

[12] D. Mercier, Minimal regularity of the solutions of some transmission problems, Math. Methods Appl. Sci. 26 (2003), 321-348.

[13] J. J. Moreau, Champs et distributions de tenseurs déformation sur un ouvert de connexité quelconque, Travaux Sém. Anal. Convexe 6 (1976), exp. 5, 23 pp.

[14] M. Petzoldt, Regularity results for Laplace interface problems in two dimensions, Z. Anal. Anwend. (2) 20 (2001), 431-455.

[15] R. T. Rockafellar, Integral functionals, normal integrands and measurable selections, in: Nonlinear Operators and the Calculus of Variations, Lecture Notes in Math. 543, Springer, Berlin, 1975, 157-207.

[16] R. Temam, Mathematical Problems in Plasticity, Gauthier-Villars, Paris, 1985.

[17] R. Temam and G. Strang, Functions of bounded deformation, Arch. Ration. Mech. Anal. 75 (1980), 7-21.

Jarosław L. Bojarski

Department of Applied Mathematics

Warsaw University of Life Sciences - SGGW

Nowoursynowska 159

02-787 Warszawa, Poland

E-mail: jaroslaw_bojarski@sggw.pl

Received on 13.1.2009;

revised version on 4.3.2011 